\title{
Materials Challenges fiom Energy to Biomaterials Forge through the 2008 Mlis Spring Meeting
}

\author{
www.mrs.org/meetings
}

The topical thread of energy wove through the 2008 Materials Research Society Spring Meeting held in San Francisco on March 24-28. For a record Spring Meeting attendance of about 4,100 participants, Meeting Chairs Jeffrey C. Gelpey (Mattson Technology), Robert J. Hamers (University of WisconsinMadison), Paul Muralt (Swiss Federal Institute of Technology EPFL), and Christine A. Orme (Lawrence Livermore National Laboratory) provided an outstanding program of 41 technical symposia and a record number of 12 tutorials, along with an equipment exhibit and various events and activities throughout the week, including recognition of the inau- gural class of 34 Materials Research Society Fellows during the Awards Ceremony on March 26. The title of MRS Fellow honors members who are notable for their distinguished research accomplishments and their outstanding contributions to the advancement of materials research worldwide.

With a full course of activities_-including Tutorials and Exhibitor Workshops - on the day before the technical sessions started, MRS named the day MegaMonday. The day opened with four lectures focusing on the critical problem of energy. MRS Bulletin had just released a special expanded issue on the topic of "Harnessing Materials for Energy." The Energy Forum

\section{Energy Forum at the 2008 MRS Spring Meeting \\ Audio and Slide Presentations \\ www.mrs.org/energyforum}

\section{OPENING REMARKS}

Presented by George W. Crabtree

Argonne National Laboratory

\section{ENERGY OVERVIEW}

Presented by George M. Whitesides

Woodford L. and Ann A. Flowers

University Professor

Harvard University, USA

Examines issues that span the technology spectrum and provides essential background to put the broad range of energy sectors in perspective.

\section{BIOFUELS and BIOMASS}

Presented by Chris Somerville

Director, Energy Biosciences Institute, and Professor, Department of Plant and Microbial Biology

University of California-Berkeley, USA

Explores the use of biofuels and biomass in the energy equation, including cellulosic ethanol and biodiesel.

\section{CATALYSIS}

Presented by Daniel G. Nocera Henry Dreyfus Professor of Energy and Professor of Chemistry Massachusetts Institute of Technology, USA

Considers the science of catalysis, with applications in fossil-fuel processes, hydrogen purification, and biofuels. Includes the synthesis of materials and structures that control chemical reactions and improve chemical properties with particular emphasis on nanomaterials.

\section{SOLAR TECHNOLOGY}

Presented by Martin A. Green

Research Director, Photovoltaic Centre

of Excellence

University of New South Wales, Australia

Investigates current limitations and new trends in solar technology, including inorganic, organic, and inorganicorganic hybrid systems. Features dyesensitized solar cells, light concentrators, and two-photon processes.

\section{CLOSING REMARKS \\ Presented by Elizabeth L. Fleischer \\ Editor - MRS Bulletin \\ Materials Research Society, USA}

was organized to complement this special issue and featured talks by scientists and researchers exploring different areas in energy. Using the metaphor of energy as a large puzzle, with various technologies forming pieces of this puzzle, the forum explored how these pieces could fit together to solve what is arguably humankind's greatest near-term challenge, namely, energy generation with minimal impact on the environment.

Delivering the opening remarks to a full house, George W. Crabtree from Argonne National Laboratory emphasized the role of materials in possible solutions to the global energy crisis. He said that global energy needs are expected to double by 2050 and triple by 2100 , driven especially by the growing energy needs of developing countries.

George M. Whitesides from Harvard University delivered the first lecture of the Energy Forum and succinctly outlined the opportunities for materials research in energy, sustainability, and global stewardship. He emphasized that issues such as energy, climate, water, and sustainability are fundamentally interconnected and that these are enormous problems with the potential to bring great conflict. Whitesides proposed the formula "well-being = available energy/ number of people" and said that there is a great need to both produce more energy and conserve energy that is currently being produced. In particular, the scarcity of water threatening several parts of the world has the potential to further exacerbate the energy crisis, as significant energy might have to be invested in water production in the future. Whitesides outlined the major sources of energy that are currently used, as well as the outlook for these energy sources over the next few decades. Fossil fuels such as hydrocarbons, coal, and gas are the mainstay of our current energy needs, but it might well be that their role in inducing deleterious climate change makes them unviable options in the very near future, he said. Furthermore, nuclear energy is plagued by concerns about proliferation and waste disposal, whereas hydroelectric power generation is currently almost at peak capacity. Solar energy represents a 
promising alternative, but very significant improvements in the design and cost of solar cells are required to harness the dilute energy of the sun. Whitesides said that, notwithstanding the claims of a hydrogen economy, it is important to realize that hydrogen is a method of transporting energy and not a source of energy.
In that sense, hydrogen is actually like electricity-it is not a solution by itself to the global energy problem.

In the short term, Whitesides continued, more research attention should be focused on the conservation of energy, such as the development of materials that reduce the tremendous power losses that occur during transmission, and the design of better illumination sources such as bright light-emitting diodes. There is also a great need to globally develop carbon management strategies to deal with the $\mathrm{CO}_{2}$ being released into the atmosphere by burning fossil fuels. Whitesides outlined several topics that he believed

\section{ACRONYM KEY}

0D: zero-dimensional

1D: one-dimensional

2D: two-dimensional

3D: three-dimensional

ACI: American Competitiveness Initiative

AFOSR: U.S. Air Force Office of Scientific

Research

AIST: National Institute of Advanced

Industrial Science and Technology

ALD: atomic layer deposition

AMD: Advanced Micro Devices

ANL: Argonne National Laboratory

ANU: Australian National University

ARO: U.S. Army Research Office

ASU: Arizona State University

ATP: adenosine triphosphate

BSO: $\mathrm{BaSnO}_{3}$

BST: barium strontium titanate

BTO: $\mathrm{BiTiO}_{3}$

BZO: $\mathrm{BaZrO}_{3}$

Caltech: California Institute of Technology

CBG: Cell Biology and Genetics

CCTO: calcium copper titanate

CMLS: Chemistry, Materials, Earth, and Life Sciences

CMOS: complementary metal oxide semiconductor

CNR-INFM: National Research Council-

National Institute for the Physics of Matter

CNT: carbon nanotube

CS: compound semiconductor

CVD: chemical vapor deposition

DMOS: double-diffused metal oxide semiconductor

DMR: Division of Materials Research

DOE: U.S. Department of Energy

DSC: dye-sensitized nanocrystalline solar cell

DVB: Digital Video Broadcasting

e-APD: electron-initiated avalanche photodiode

EC: electrochemical capacitor

EES: electrical energy storage

EFRC: Energy Frontier Research Center

EPFL: École Polytechnique Fédérale de Lausanne

FeRAM: ferroelectric random-access memory

FET: field-effect transistor

FinFET: fin field-effect transistor
Georgia Tech: Georgia Institute of

Technology

GPS: Global Positioning System

GSM: Global System for Mobile communications

HEMT: high-electron-mobility transistor HTS: high-temperature superconductor

IBAD: ion-beam-assisted deposition

IC: integrated circuit

IGBT: insulated gate bipolar transistor

IMEC: Illinois Manufacturing Extension Center

IR: infrared

JHU: Johns Hopkins University

LAAS: Laboratory for Analysis and Architecture Systems

LANL: Los Alamos National Laboratory

LBNL: Lawrence Berkeley National Laboratory

LED: light-emitting diode

LEDS: low-energy dislocation structure

LIDAR: light detection and ranging

LLNL: Lawrence Livermore National Laboratory

MATIS: Center for Materials and

Technologies for Information and

Communication Science

MBE: molecular beam epitaxy

MEMS: microelectromechanical systems

MFP: mean free path

MIT: Massachusetts Institute of Technology

MOCVD: metal-organic chemical vapor deposition

MOS: metal oxide semiconductor

MPI: Max Planck Institute

MS\&E: Materials Science \& Engineering

NASA: U.S. National Aeronautics and Space Administration

NCSU: North Carolina State University

NEC: Nippon Electric Company

NMR: nuclear magnetic resonance

NNIN: National Nanotechnology

Infrastructure Network

NREL: U.S. National Renewable Energy Laboratory

NRL: U.S. Naval Research Laboratory

NSF: U.S. National Science Foundation

NVM: nonvolatile memory

OFET: organic field-effect transistor

OLED: organic light-emitting diode

ONR: U.S. Office of Naval Research
ORNL: Oak Ridge National Laboratory

PCBM: [6,6]-phenyl-C61-butyric acid methyl ester

PCDFB: photonic crystal distributed feedback

PCPDTBT: poly[2,6-(4,4-bis-

(2-ethylhexyl)- $4 H$-cyclopenta

[2,1-b;3,4-6']-dithiophene)-alt-4,7-

(2,1,3-benzothiadiazole)]

PLD: pulsed laser deposition

PSI: Paul Scherrer Institute

PSU: Pennsylvania State University

PV: photovoltaic

PZT: lead zirconate titanate

R\&D: research and development

REBCO: rare-earth-barium-copper-oxide rf: radio frequency

RFID: radio-frequency identification rf-SoP: radio-frequency-system on package RPI: Rensselaer Polytechnic Institute

SAM: self-assembled monolayer

SBH: Schottky barrier height

SBL: supported bilayer

SBT: strontium bismuth tiatanate

SNL: Sandia National Laboratories

SOI: silicon-on-insulator

SPER: solid-phase epitaxial regrowth

STO: strontium titanate

SUNY: State University of New York SWNT: single-walled carbon nanotube

TCO: transparent conducting oxide

TEM: transmission electron microscopy

TFT: thin-film transistor

TU: Technische Universiteit

UC: University of California

UCLA: University of California-Los Angeles

UCSB: University of CaliforniaSanta Barbara

UFRGS: Federal University of Rio

Grande do Sul

UIUC: University of Illinois at UrbanaChampaign

UNSW: University of New South Wales

UT: University of Texas

UV: ultraviolet

VCSEL: vertical-cavity surface-emitting laser

WiMAX: Worldwide Interoperability for Microwave Access

ZT: dimensionless thermoelectric figure of merit 
are fertile grounds for the next generation of materials researchers; these include catalysis, separations, materials with extreme properties, approaches that enable more efficient movement of electrons in matter, scaling to nanoscale dimensions, and systems capable of matching the complexity of biological processes such as photosynthesis. Given the current corporate atmosphere that emphasizes short-term returns, Whitesides said that this effort will have to be led by research universities, perhaps in collaboration with venture philanthropic organizations and local and federal government.

"The good news is that this is an issue that the public deeply cares about in one form or the other," said Whitesides; "the problem is that even if we apply all that we know, we still come up short." He added that, because the basic knowledge does not exist, we have no alternative but to create new knowledge to solve these problems.

Following Whitesides's overview, Chris Somerville of the University of CaliforniaBerkeley discussed the area of biofuels, emphasizing the importance of converting cellulose to ethanol and other fuels such as syngas and diesel. Through replacing grain-based fuels by cellulosic fuels, biofuels would be disassociated from the food chain, their net energy efficiency would be increased, and their energy capacity would be dramatically enhanced.

Daniel G. Nocera of the Massachusetts Institute of Technology spoke about the broad field of catalysis. He proposed that an alternative sustainable energy source with the capacity to replace dependence on fossil fuels is to split water to make hydrogen by using sunlight. Biology provides the proof of principle, he said, pointing to green plants that split water at room temperature with high efficiency. Nocera described chemical approaches using inexpensive catalysts for accomplishing the same feat, making use of the lessons from nature.

Martin A. Green of the University of New South Wales, Australia, provided a comprehensive overview on the current and future opportunities of photovoltaics (PVs), as well as predictions and new trends in solar technology including inorganic, organic, and hybrid systems. He specifically discussed the need for third-

\section{Materials Research Society BLOGS

generation photocells that will have much higher efficiency and lower cost than the first- (bulk materials) and secondgeneration (thin films) to drive widespread penetration of solar energy. Possibilities for the third-generation cells include hot carrier systems, thermal PV, thermionics, quantum dot systems, and interband converters. Audio and slide presentations of the Energy Forum are available at Web site www.mrs.org/ energyforum.

The Plenary Session capped MegaMonday, highlighting the presentation by Michael Graetzel of École Polytechnique Fédérale de Lausanne, Switzerland, who addressed solar power. Although most of the research focus has been on solid-state photovoltaics, there is a new paradigm, he said-mesoscopic solar cells based on interpenetrating network junctions. These include dye-sensitized nanocrystalline solar cells (DSCs), which can achieve a quantitative conversion of photons into electric current. The workhorse dye used is a ruthenium-based dye. Currently, the solar-to-electric power efficiency has been demonstrated to be above $11 \%$-in particular, DSCs outperform silicon at low light levels. Graetzel said that charge separation in DSCs is by kinetic competition similar to natural photosynthesis. The efficiencies of DSCs can still be vastly improved according to theoretical predictions, he said. Among examples of various materials that can be used for energy harvesting and conversion and that are under investigation are advanced nanostructures, new sensitizers, and quantumdot-sensitized cells. Solar cells with different colors, aesthetically pleasing, can now be produced, and a commercial production plant for DSCs is in operation in Cardiff, Wales; its first product is a lightweight power supply for mobile phones.

William F. Banholzer, Executive Vice President and Chief Technology Officer of The Dow Chemical Company, contributed to the focus on energy in his overview presentation in Symposium X. The present changing energy scenario is affecting the chemical industry, he said, as its foundation was built upon the use of coal and natural products as feedstocks. Over decades, the industry migrated to using oil and natural gas as its primary sources of carbon because of straightforward cost optimization. Natural gas liquids and oil are more reduced forms of carbon with structures similar to those of the desired end products. Use of these feedstocks allows the petrochemical industry to move downhill in enthalpy (oxidation), maximizing carbon utilization and minimizing the capital required for plants and equipment. Mas- sive inflation in energy costs and increased price volatility have challenged the current paradigm, Banholzer said. He explained that the U.S. chemical industry is now disadvantaged relative to most of the world and has lost its ability to export products. In addition, the increasing concern over greenhouse gas emissions has added a new dimension to the energy debate. Renewable feedstocks are garnering publicity as alternatives to fossil fuels. Although there will be some niche applications for bioderived feedstocks, it is unlikely that there will be a significant transformation of the chemical industry for many decades, Banholzer said. According to Banholzer, photosynthesis is fundamentally a poor energy conversion process, converting much less than $1 \%$ of the incident solar energy into chemical energy. This poor energy conversion efficiency means that very large land areas are required to produce any appreciable amount of material. He cited as an example the world's largest bioderived chemicals plant, which is being built in Brazil by Dow and partner Crystalsev. This plant will use sugar cane to produce 350,000 tons of polyethylene. Such a plant is economically viable only in tropical regions, and it will still require 120,000 hectares of land. If the world's global ethylene supply were to be produced from sugar cane, it would require a land mass equal to the entire state of California, and ultimately, the chemical industry must compete with the food and transportation fuels sectors for land use.

Even if adequate land were allocated to generate chemical feedstocks, Banholzer said, the capital required to replace the current infrastructure would be tremendous. Great hope is placed on cellulosic ethanol to reduce land requirements. However, even if it could be implemented using the most idealized process flows envisioned, approximately $\$ 400$ billion in new plants would be required just to replace current ethylene production. Moreover, ethylene from ethanol is the best case as dehydration is a low-cost, high-yield process, he said. While being the single largest chemical feedstock, ethylene still represents only $25 \%$ of input materials to the chemical industry. A figure like $\$ 400$ billion is a massive number when one recognizes that the total annual capital spending of the top 50 chemical companies combined is less than $\$ 40$ billion, he said. Current production costs-when compared to other alternatives-do not produce sufficient returns to motivate this expenditure. Ethanol in the United States would have to sell for $\$ 0.15$ per gallon to be on a par with Middle East ethane. 
Coal is another potential carbon source. It is a relatively cheap source of carbon, and on an energy basis, it is the lowest cost feedstock, said Banholzer. However, coal is also more oxidized than most chemical products, requiring a reducing agent or some source of energy to allow it to be used as a chemical feedstock. The most common way this need is addressed is through gasification-the energy in coal is reapportioned so that some carbon is reduced and the rest oxidized to $\mathrm{CO}_{2}$. This approach requires large capital for the gasification plants and releases significantly more $\mathrm{CO}_{2}$ than other processes. Banholzer said that photovoltaic (PV) cells are at least an order of magnitude more efficient than photosynthesis at converting sunlight into electricity. The problem with $\mathrm{PV}$, he said, is that it is currently too expensive, making cost reduction a major opportunity for the future.

\section{Energy and Environment}

In keeping with the strong focus on energy at the MRS meeting, L. Hammarstrom (Uppsala Univ., Sweden) discussed photobiological approaches to the production of energy from sunlight and water. Delivering an invited lecture in Symposium LL, Hammarstrom said that PV alone does not constitute a solution to the energy crisis, but that a means of storing energy such as hydrogen is required. Several approaches for hydrogen production are based broadly on mimicking or harnessing photosynthetic processes. The first set of approaches involves the production of hydrogen by heterocyst cyanobacteria in an ambient oxygen atmosphere. The genetic modification of these bacteria by the introduction of foreign hydrogenases appears to be a promising approach to improve efficiency, Hammarstrom said. Although photosynthesis as a whole is not a very efficient process, each of the primary steps is very efficient. Thus, an alternative approach involves the use of biomimetic complexes that are optimized for single-step energy conversion. Hammarstrom and his colleagues have designed several such complexes based on manganese centers with tyrosine-like ligands to oxidize water. These complexes are additionally equipped with rutheniumbased sensitizers to harvest light. Such bioinspired synthetic reaction centers offer an interesting alternative to conventional heterogenous catalysts, he said.

In Symposium KK, A. Freundlich (Univ. of Houston) discussed the use of quantum confinement to improve efficiency in solar cells. This involves the creation of intermediate states within the bandgap of

\section{Graduate Students Receive Gold and Silver Awards}

Graduate Student Awards were announced during an evening ceremony on March 26 at the 2008 Materials Research Society Spring Meeting in San Francisco.

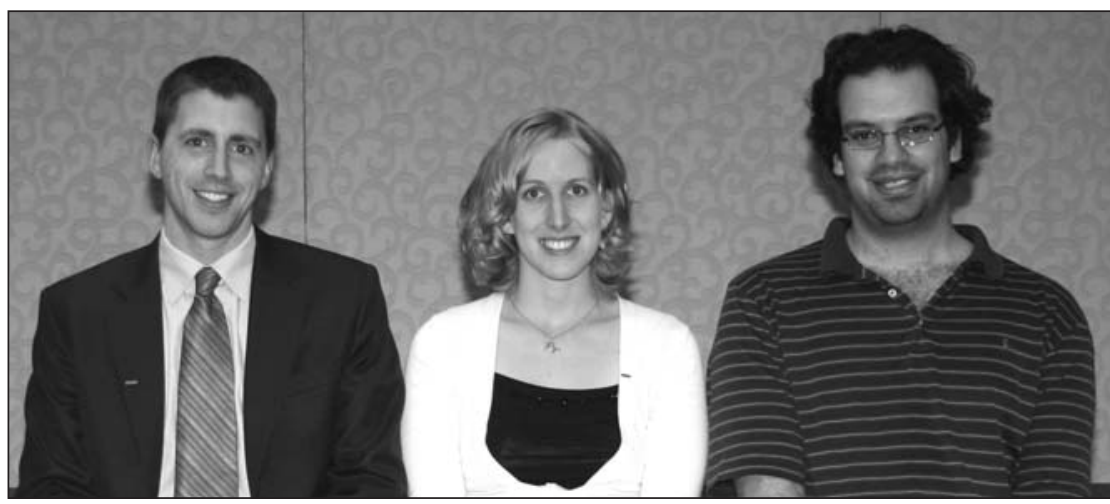

Gold Graduate Student Awards were awarded to (left to right): David A. Giljohann (Northwestern University), Jennifer A. Dionne (California Institute of Technology), and Akram I. Boukai (California Institute of Technology).

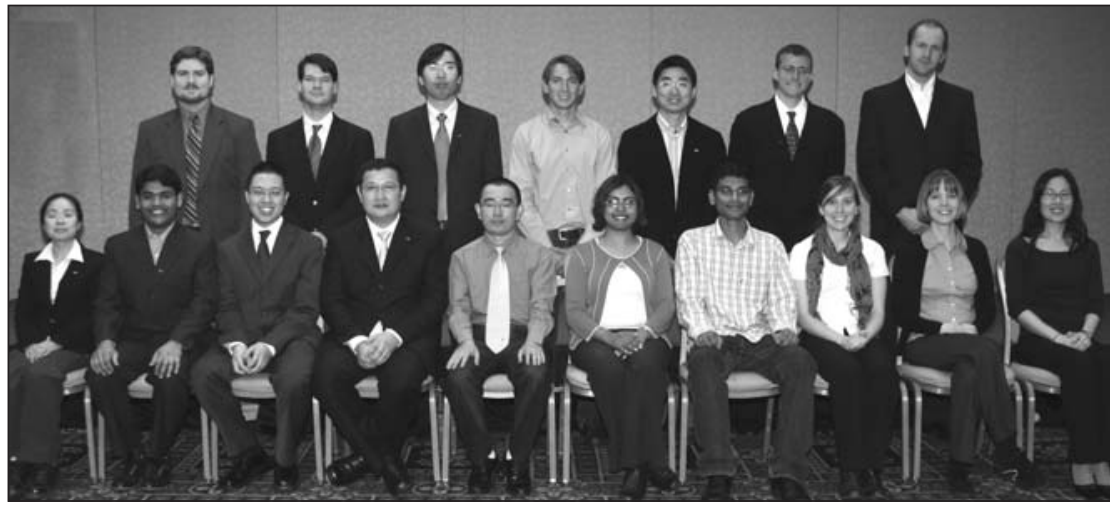

Silver Graduate Student Awards were awarded to (front row, left to right): Shenyuan Yang (Chinese Academy of Sciences and The University of Tennessee), Ramanathaswamy Pandian (University of Groningen), lan Y. Wong (Stanford University), Nurxat Nuraje (The City University of New York), Jun Lu (University of Utah), Kalapi G. Biswas (Purdue University), Muralidhar Ambati (University of California-Berkeley), Alexis D. Ostrowski (University of California-Santa Barbara), Melissa J. Archer (California Institute of Technology), and Judy J. Cha (Cornell University); and (back row, left to right): Andrew L. Schmitt (University of WisconsinMadison), Jeffrey Peet (University of California-Santa Barbara), Feng Jiao (University of St. Andrews), Eric D. Pressly (University of California-Santa Barbara), Jian Shen (University of California-San Diego), Khalid M. Hattar (University of Illinois, UrbanaChampaign), and Brendan T. O'Connor (University of Michigan). Not shown is Susan E. Habas (University of California-Berkeley).

the $p-n$ diode by incorporating lowdimensional semiconductor structures (e.g., wells, dots, wires). Acknowledging that improved efficiencies have not yet been achieved by quantum confinement, Freundlich provided an overview of the problems that arise, such as difficulty in carrier extraction and low density of defect states. A. Marti (Univ. Politécnica de Madrid) expounded on some of these issues, specifically in a quantum-dot PV device where poor efficiency for intermediate-to-conduction band transitions might actually lower the overall cell efficiency. However, a significant increase in efficiency over conventional cells is possible if light trapping and light concentration are incorporated, Marti said. H. Stiebig (IPV, FZ Jülich, Germany) described approaches for trapping light in 
PVs in order to improve efficiency, using textured back-reflectors designed to maximize the path length of photons in the device. Methods discussed included surface roughness, periodic rectangular gratings, and stepped pyramid-like structures, all textured on a reflective back surface, as well as silver nanoparticles sandwiched between the $p-i-n$ junction and a transparent oxide layer. Stiebig presented optimal parameters for the various techniques, such as the ideal step height and period for the pyramids and an ideal size of $25 \mathrm{~nm}$ for the silver particles.

The U.S. Department of Energy recently held a workshop on basic research needs for electrical energy storage. In Symposium JJ, A.A. Gewirth (UIUC) reported

\section{Poster Prizes Awarded at 2008 MRS Spring Meeting}

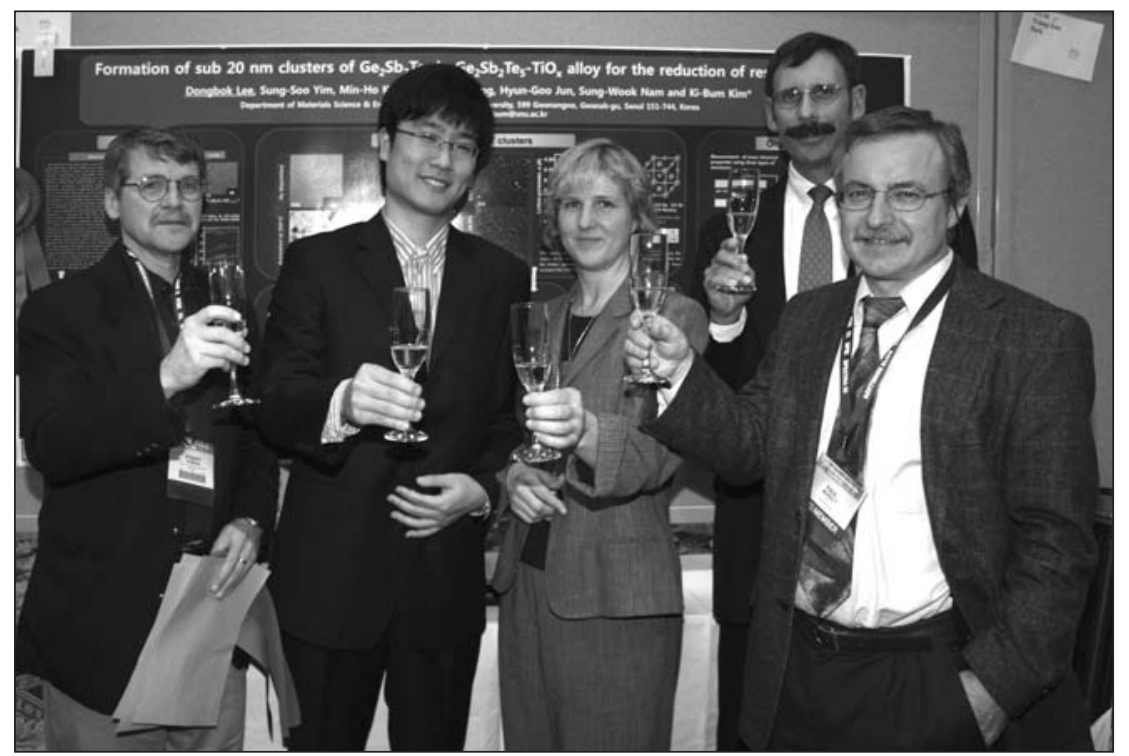

The 2008 Spring Meeting Chairs awarded prizes for the following best poster presentations:

(F3.6) Polarization of Strained $\mathrm{SrTiO}_{3}$ Films Grown on $\mathrm{Si}(001)$ and the Ferroelectric FET, F. Walker, Y. Segal, J. Reiner, and C. Ahn (Yale Univ.) and Z. Zhang (ANL); (F9.1) Molecular Conductance Switch-On of Single Ruthenium Complex Molecules, K. Seo , A.V. Konchenko, J. Lee, G.S. Bang, and H. Lee (Electronics and Telecommunications Research Institute, South Korea); (G3.15) Formation of Sub-20-nm Cluster of $\mathrm{Ge}_{2} \mathrm{Sb}_{2} \mathrm{Te}_{5}$ in $\mathrm{Ge}_{2} \mathrm{Sb}_{2} \mathrm{Te}_{5}-\mathrm{TiO}_{2}$ Alloy for the Reduction of Reset Current in Phase Change Random Access Memory, D. Lee, S.-S. Yim, M.-H. Kwon, D. Kang, H.-G. Jun, S.-W. Nam, and K.-B. Kim (Seoul National Univ., South Korea); (I5.28) Magnetic Properties of ZnO Nanoparticles, A. Quesada, M.A. Garcia, A. Hernando, J.M. Merino, E.F. Pinel, J. de la Venta, J. Llopis, M.L. Ruiz Gonzalez, and J. Gonzalez Calbet (Universidad Complutense de Madrid, Spain) and G. Castro (European Synchrotron Radiation Facility, Grenoble, France); (O4.13) Ferromagnetic Semiconducting $\mathbf{F e}_{1_{-x}} \mathrm{Co}_{x} \mathrm{Si}$ Alloy Nanowires: Building Blocks for Silicon Based Spintronics, A.L. Schmitt, J.M. Higgins, and S. Jin (Univ. of Wisconsin-Madison); (P8.12) Size-Dependence Peculiarities of Supported Small Clusters and Their Effect on Single-Walled Carbon Nanotubes Growth, O.A. Kuznetsov, E. Pigos, G. Chen, and A.R. Harutyunyan (Honda Research Institute USA Inc.) and T. Tokune (Honda R\&D Co. Ltd., Japan); (T5.13) Using Scanning Probe Microscopy to Probe Nanoscale Electric Field Fluctuations, S. Yazdanian, S. Kuehn, and J. Marohn (Cornell Univ.); (AA11.34) HighPerformance Single-Crystal Field-Effect Transistors for the Probing of Charge Transport in Organic Materials, C. Reese, W.-J. Chung, S.C.B. Mannsfeld, R. Stoltenberg, M. Roberts, and Z. Bao (Stanford Univ.); and (NN7.13) Uranium Complexes Supported by Redox Active Ligands: Electronic Structure, Bonding and Reactivity, E. Schelter, B.L. Scott, J.D. Thompson, D.E. Morris, and J.L. Kiplinger (LANL). that the workshop identified several principal research directions for developing electrical energy storage systems that combine safety, high storage capacity, long lifetime, and low cost. Gewirth said that it is critical to probe energy storage chemistry and physics at all time and length scales and to develop novel techniques to study the dynamics and structures of these systems. Furthermore, understanding the efficacy of structure in energy storage is of great importance. Gewirth also emphasized the need to study charge transfer and transport in energy storage systems and to develop innovative electrical energy storage electrolytes based on either polymers or ionic liquids. The final principal research direction agreed upon in the workshop calls for emphasis on multiscale computation for electrical energy storage. Gewirth said that the primacy of electrochemistry in solving future energy needs has become clear, but the funding climate is cause for much concern.

In the area of potential environmental risks posed by nanomaterials, V. Colvin (Rice) addressed how the uncertainty in possible effects of nanomaterials on people and the environment is turning out to be a barrier to the application of nanomaterials in real-world applications. Colvin presented results from the Center for Biological and Environmental Nanotechnology at Rice University, which is an NSF-funded center that focuses on studying interactions at the wet-dry interface between inorganic nanomaterials and water or biological entities. Given the many different parameters that are varied for nanomaterials, conventional toxicology studies of a single type of nanomaterial are not going to be very instructive, she said. Instead, Colvin explained that there is a need to understand structure-function relationships and to develop predictive models for the biological impact of nanomaterials. For example, studies at Rice illustrate the very different in vitro cytotoxicities of variously functionalized fullerenes and SWNTs. The latter species tend to deposit on cell membranes but have relatively lower toxicities than fullerenes. Colvin concluded by presenting results of the poly(ethylene glycol) coating of nanoparticles to prevent interactions with proteins. Surprisingly, much of the wisdom regarding antifouling coatings for 2D surfaces does not hold true for 3D nanomaterials, she said.

In another presentation on the environmental impacts of nanoscale materials, M. Wiesner (Duke) outlined the physical and chemical factors controlling the expo- 
sure, transformation, and reactivity of fullerene nanomaterials. In a presentation in Symposium MM, Wiesner said that the partitioning of nanomaterials in environmental systems is of key importance. $\mathrm{C}_{60}$ fullerenes by themselves have very low solubility in water, but $\mathrm{C}_{60}$ aggregates stabilized by solvents or natural organic matter such as humic acids and polysaccharides have the potential to be transported through various media. Wiesner said that, although previous studies of the toxicity of $\mathrm{C}_{60}$ have emphasized the role of parameters such as surface derivatization, it is the aggregation state and size of the aggregates that play a far more important role. In light of the new data, Wiesner and his colleagues believe that the toxicity of fullerenes might actually originate from particle-induced oxidative stress within cells and not from the production of reactive oxygen species.

\section{Electronics, Magnetics, and Photonics}

In a discussion about production technology for solar-based green energy, M. Shimosawa (Fuji Electric Systems Co., Japan) introduced Fuji's production technology of large-area, lightweight $\left(8 \mathrm{~kg} / \mathrm{m}^{2}\right)$, flexible solar cells and modules. The a-Si/a-SiGe-based thin-film solar cells consist of a unique monolithic device structure using through-hole contacts and are manufactured on flexible plastic films using a continuous process. The technology consists of a low-temperature and high-speed deposition method for textured electrodes and a high-speed deposition method for amorphous silicon, a modified roll-to-roll deposition apparatus technology, a large-area high-quality transparent electrode deposition, and a very high-speed laser patterning method, Shimosawa explained in Symposium A. To scale up production capacity, a lowtemperature process with a higher deposition rate than before for the textured metal electrodes, termed "selective reactive sputtering," was developed and implemented. This deposition method utilizes an $\mathrm{Ar} / \mathrm{O}_{2}$ gas mixture for sputtering an $\mathrm{Ag}$ alloy target containing a small amount of Al. The silver is not oxidized and maintains its low resistivity, whereas the $\mathrm{Al}$ is oxidized selectively and changes the surface morphology dramatically. The oxide incorporation affects the surface reactions and enhances the formation of a rougher surface.

Furthermore, Fuji developed a deposition control method for amorphous silicon that allows for a controlled increase in deposition rate without deterioration of film properties. The researchers found that the peak-to-peak voltage at the cathode
$\left(V_{\mathrm{pp}}\right)$ had an influence on the plasma and could be used for optimizing the deposition conditions. $V_{\mathrm{pp}}$ is closely related to the long-term stability of the solar cell efficiency. Also, a high-speed laser patterning method was developed that uses a galvano scanner, resulting in an improved patterning speed. The new production facility in Kumamoto, Japan, went online last year; it produces $12 \mathrm{MW} /$ year and can process 1-m-wide and 2,000-m-long film substrates. Shimosawa said that Fuji plans to install more production lines to ramp up production to $40 \mathrm{MW} /$ year capacity in fiscal year 2008. This type of low-cost mass production is needed to make solar cell technology more affordable for widespread implementation. However, the efficiency of just $7 \%$ leaves much to be desired. Still, this advanced process technology is considered crucial for efficient large-scale and low-cost manufacture of future much improved cell technologies, said Shimosawa.

To meet increasingly challenging and complex system requirements and to remain cost-effective, it is not enough to use one single semiconductor materials system. Therefore, major efforts have been expended in recent years to combine the low-cost and well-established Si-based CMOS processing attributes with the superior performance attributes of compound semiconductors (CSs). Such a combination will enable performance superior to that achievable with CS or CMOS technology alone, but with CMOS affordability. With an approach that directly integrates the CS into the CMOS wafer, only one wafer is processed to achieve a finished chip. Therefore, great efforts have been made to achieve direct integration of III-V materials systems such as $\mathrm{GaN}$ and $\mathrm{GaAs}$ with Si. In Symposium C, J. Derluyn (IMEC, Belgium) reported on crack-free AlGaN/ GaN HEMT devices grown on 6-in. Si substrates with low sheet resistance. The researchers used in situ SiN passivation, which solved the dispersion problem, and avoided the relaxation and cracking of the AlGaN layer by freezing in $\mathrm{Ga}$ (inhibiting mobility), leading to a strained AlGaN layer without cracking. In one case, a breakdown voltage of over $600 \mathrm{~V}$ was achieved. The devices were grown on 6-in. $\mathrm{Si}\langle 111\rangle$ substrates and passivated. The epitaxial growth was optimized for minimum rf losses achieved with the introduction of the substrate-nitride interface while at the same time maintaining high buffer resistivity and low trap density. To assess rf losses of the epitaxial layer structure, coplanar waveguides were defined at sites of the wafer where the top in situ $\mathrm{SiN}$ and $\mathrm{AlGaN}$ has been etched away.
The attenuation of the rf signals on the coplanar waveguides remained below $0.3 \mathrm{~dB} / \mathrm{mm}$ for frequencies of up to $6 \mathrm{GHz}$. The devices' rf power performance was characterized on-wafer (worst-case scenario because of severe thermal limitations). The researchers observed that the maximum gate current remained below $50 \mu \mathrm{A} / \mathrm{mm}$ even at power density levels of $7.9 \mathrm{~W} / \mathrm{mm}$, which promises very good reliability. The larger 20-finger devices of $5-\mathrm{mm}$ total gate periphery reached a maximum absolute output power of $20 \mathrm{~W}$ at a bias of $40 \mathrm{~V}$, which represents the limit of IMEC's on-wafer measurement system.

A number of presentations in Symposia $\mathrm{I}$ and $\mathrm{F}$ focused on understanding and exploiting the influence of the substrate on ferroelectrics. In Symposium I, C.A. Vaz (Yale) presented results on the effect of a $\mathrm{BiTiO}_{3}$ (BTO) substrate on $\mathrm{Fe}_{3} \mathrm{O}_{4}$. Grown at the large strain of $5.2 \%$, a sizable variation was seen in the magnetic anisotropy of the $\mathrm{Fe}_{3} \mathrm{O}_{4}$ as the BTO underwent phase transformations with decreasing temperature. Because BTO is a piezoelectric, controlling its phase with an electric field holds the promise of accessing magnetoelectric coupling in this system.

Similar topics arose in Symposium F, where R. Ramesh (UC-Berkeley) presented an overview of work on $\mathrm{BiFeO}_{3}(\mathrm{BFO})$, a candidate ferroelectric to replace leadbased lead zirconate titanate (PZT). He said that it shows great promise for use in nonvolatile memory applications, with larger polarizations than the current workhorse ferroelectrics (PZT and barium stron-

\section{Winners of the MRS Entrepreneurship Challenge Announced}

In the 2008 MRS Entrepreneurship Challenge, the grand prize went to Romit Dhar (Technical Lead) and Avimanyu Datta (Business Lead), both of Washington State University, for their business plan entitled, "Cost-Effective Single-Crystal Piezoelectric Devices for Energy Harvesting and Transduction." The runners-up were Scott Bridgeman (Technical Lead) and Jitendra Kumar (Business Lead), both of the Ohio State University, for "Biodegradable Polymer for Increased Cell Proliferation Rates"; and Technical Lead Mihaela Jekic of the Ohio State University and Business Lead Bruce Caldwell of the University of Cincinnati for "Nanosize Gene and Drug Delivery Device Propels Research." 
tium titanate, i.e., BST), very low fatigue, and minimal imprint on switching. The polar domain retention at high temperatures is excellent, he said, and shows no dramatic loss of domain orientation after $50 \mathrm{~h}$ at $500^{\circ} \mathrm{C}$. Ferroelectric behavior was even seen in films on the order of a few unit cells thick, but Ramesh warned that such results should be taken with caution given the great difficulty of quantitative measurement of order parameters on these scales, and because BFO films currently exhibit larger leakage and coercive fields than PZT and strontium-bismuth-tiatanate (SBT), which are the two ferroelectric materials in smart cards with nonvolatile ferrolectric memories in the current market. He identified the main challenges in the field as understanding imprint, fatigue, and leakage before BFO can be technologically viable, but confessed to being distracted himself by the tantalizing prospect of multiferroic coupling in this material.

Another approach to achieving this

\section{The Nabarro Legacy Celebrated at 2008 MRS Spring Meeting}

It is hard to overstate the impact of Frank Nabarro's contributions to modern-day materials science and engineering. His seminal research on dislocation theory has had a profound impact on numerous present-day materials and processes. The Peierls-Nabarro force on moving dislocations and the Herring-Nabarro creep theory will keep his name in materials science textbooks for generations to come. He has made major contributions on the elastic theory of dislocations, the theory of work hardening, the effect of elastic energy on the shape of precipitates, diffusional creep, the mechanism of rafting in superalloys, and dislocation patterning. Symposium $Z$ was designed to honor Nabarro's legacy in the field and included an all-day series of invited talks by experts in various disparate areas touched by his discoveries.

Among the speakers was G. Dehm (Montanuniversität Leoben, Leoben, Austria), who described dislocations and plasticity in the deformation of very small metallic structures. This is hardly an academic exercise, as such metallic structures are found in various applications, including microelectronics and MEMS devices. Dehm first described straining of polycrystalline copper wires using a specially designed setup including tweezers. In situ tensile testing of wires with diameters between $50 \mu \mathrm{m}$ and $0.5 \mu \mathrm{m}$ and of films with thicknesses down to $40 \mathrm{~nm}$ was carried out. He then discussed size effects in microcompression testing demonstrating dislocation pileup at the flat punch interface, particularly at small aspect ratios, resulting in a significant increase in flow stress. This likely contributes to the observed size effect. Dehm also described the in situ tensile testing of 40-nm-thick single-crystal Au films revealing dislocation-driven plasticity. However, deformation was found to be carried by partial dislocations rather than perfect dislocations, in contrast to the case for film thicknesses above ca. $100 \mathrm{~nm}$.

D. Kuhlmann-Wilsdorf (Univ. of Virginia) knew Nabarro well and worked with him for several years. Most of her talk was devoted to reminiscences of the circumstances leading to her working with Nabarro. She met him first at a conference just after World War II. It was clearly a difficult time to be a female German scientist and researcher at this time, and Kuhlmann-Wilsdorf described how she and her work were often not taken seriously. She moved to Bristol, England, to work with Nabarro and actually stayed three months with Nabarro and his wife in a room sublet from them. She then got married and moved to South Africa. Subsequently, Nabarro also moved to South Africa and became her Departmental Chair at the University of the Witwatersrand. He very successfully developed the department. Kuhlmann-Wilsdorf eventually moved to the United States but continued to work in areas related to Nabarro's work.

Kuhlmann-Wilsdorf devoted the rest of her talk to describing the development of a theory of low-energy dislocation structures (LEDSs) that explains the workhardening behavior of materials. The LEDS theory asserts that, while under stress, all parts of deformed materials are in mechanical equilibrium everywhere and have the lowest free energy achievable under the prevailing conditions of friction. Release of the stress then causes only minor rearrangements of the crystal defects. She said that the LEDS theory has already quantitatively explained the bulk of all important aspects of plasticity and their correlated dislocation structures, for both "planar" and "wavy glide" metals, in unidirectional strain and in fatigue, and for single crystals and polycrystals.

Presentations will be published in MRS Proceedings Volume 1090E. The symposium received support from Brucker AXS, Inc.; FEI Co.; Hysitron; JEOL USA, Inc.; LLNL; NSF; ONR; and Veeco Metrology, Inc. coupling is the use of multiphase heterostructures, as shown by A. Gupta (Univ. of Alabama) in Symposium I. In this case, however, Gupta sought to remove or decrease the influence of the substrate by using arrays of $\mathrm{MgO}$ nanowires, which exhibit minimal clamping to the substrate. The nanowires were synthesized by a CVD process in which gold nanodots were first patterned onto an $\mathrm{MgO}$ substrate to seed the growth of vertical $\mathrm{MgO}$ nanowires. The wires were cubic rods rather than columns and grown in a dense forest, pushing the gold dots up off the substrate as they grew. Gupta also demonstrated manipulation of single wires, placing them across electrodes with a microprobe to measure their individual electric and magnetoelectric properties.

"Are all nanoparticles magnetic?" asked A. Sundaresan (Jawaharlal Nehru Center for Advanced Scientific Research, India), delivering an invited lecture in Symposium I. Conventional wisdom holds that, because magnetism is associated with the presence of unpaired electrons, materials that do not have unpaired electrons are not expected to be magnetic. However, Sundaresan and his collaborators have found that, upon shrinking nonmagnetic oxides such as $\mathrm{CeO}_{2}, \mathrm{Al}_{2} \mathrm{O}_{3}$, and $\mathrm{ZnO}$ to nanoscale dimensions, these materials start to exhibit significant magnetization behavior. Sundaresan said that surface point defects, either oxygen deficiencies or cation vacancies, give rise to surface ferromagnetic behavior. In other words, the nanoparticles can be thought to have a diamagnetic core with a ferromagnetic shell. Sundaresan said that these results are also generalizable to nitrides and chalcogenides, suggesting the possibility of the existence of a new class of universal magnetic materials. Sundaresan and his colleagues have also examined $\mathrm{BaTiO}_{3}$ nanoparticles, where the presence of surface ferromagnetism implies that a ferroelectric shell is surrounded by a ferromagnetic core, pointing to the possibility of engineering functional multiferroic materials.

\section{Nanomaterials, Fundamentals, and Characterization}

When silicon reaches its limits of scaling in electronic devices, can carbon step in? Recent research on highly conductive CNTs and then graphene sheets indicates the potential. In Symposium P, W.A. de Heer (Georgia Tech) presented the potential to massively scale up fabrication based on graphene multilayers grown epitaxially on single-crystal silicon carbide. Unlike exfoliated graphene, epitaxial graphene is grown on macroscopic wafers, and devices can be patterned in a 
continuous film over the entire surface with high reliability. The patterning features resemble nanotubes in their behavior, with the graphene bandgap in electron volts on the order of $1 / \mathrm{w}$, where $\mathrm{w}$ is the width in nanometers. De Heer described the discovery of the bandgap on graphene grown on the silicon-terminated face of silicon carbide and of an unusual rotated phase of multilayered graphene grown on the carbon-terminated face. This causes the electronic band structure (and the electronic properties) to be similar to that of single-layer graphene rather than that of graphite, he said. Wires, loops, and ribbons can be patterned, and several fieldeffect devices have been made. Although, at present, they make "bad" transistors, the electrical characteristics are very consistent over many samples, opening the way to reproducible improvements. The presentation also showed evidence for Schottky barrier behavior for graphene oxides, which can then be converted to graphene through heating or electronbeam irradiation. These recent developments further amplify the feasibility of patterned epitaxial graphene for nanoelectronics, de Heer said.

Nanostructures are finding increasing use in biomedical engineering for both diagnostic and therapeutic purposes. Y. Xia (Washington Univ., St. Louis) discussed the use of gold nanostructures to improve contrast in optical coherence tomography, which is a promising "optical biopsy" method for early cancer detection. Delivering an invited lecture in Symposium V, Xia said that, for most biological applications, optically active nanostructures have to be tuned to absorb light within a very narrow window so as not to overlap with the strong absorptions of hemoglobin and water. Fortuitously, the surface plasmon resonance of gold nanostructures can be tuned from the blue to the near-infrared regions of the electromagnetic spectrum, and thus, these materials can be used as contrast agents for various tissue-imaging techniques. Xia and his colleagues have developed a method to fabricate gold nanocage structures based on the galvanic replacement of a sacrificial silver nanocube template. The optical absorption of these structures can be tuned by adjusting the thickness of the nanocage walls. These nanostructures have been functionalized with specific antibodies that allow them to bind to cancer cells. The specific binding of these nanostructures to cancer cells allows the differentiation of cancer cells from regular cells during optical coherence tomography. The gold nanocages have also been used to enhance contrast in photoacoustic tomography, thus enabling the accurate mapping of blood vessels in the brain of a living rat, Xia said.

L. Samuelson (Lund Univ., Sweden) presented an overview of recent advances in the growth and device integration of epitaxial III-V nanowires. Delivering an invited lecture in Symposium O, Samuelson said that semiconductor nanowires represent an attractive bottom-up approach to devices at scales where quantum confinement becomes important, and will likely play a very important role in the transistor scaling roadmap over the next several decades. The nanowires are grown by the catalytic action of gold nanoparticles on different substrates and can be patterned to form ordered arrays and heterostructures. Samuelson discussed some recent results on zinc blende/wurtzite twinning in InAs nanowires, which leads to the formation of kinks and variations in optical properties. The formation of banded InP/InAs heterostructured nanowires enables observation of interesting quantum effects such as 1D steeplechase of electrons, single-electron pumping, and singleelectron transistors. Samuelson concluded with some examples of the application of core-shell GaAs/GaInP nanowires in LEDs. Samuelson said that nanowires can now be looked upon as generic enabling device platforms for applications ranging from spintronics to solid-state lighting.

\section{Polymers and Biomaterials}

The functioning of the world's smallest rotary motor was outlined by G. Oster (UC-Berkeley) in Symposium FF. Indeed, the world's smallest rotary motor is not an object that is nanofabricated by engineers but is ATP synthase, a protein devised by nature. The protein has two reversible rotary motors: FO, which functions as an ion turbine or pump and is embedded within a membrane, and F1, which functions as a hydrolysis motor or an ATP synthesizer and is soluble. The two parts are connected together by a shaft and operate according to distinct principles. Theoretical work by Oster and his colleagues, along with input from experimentalists, has allowed reconstruction of the approximate kinematic motion of the two segments. The protein pump is remarkably versatile and reversible. The flow of protons and sodium ions drives ATP synthesis, whereas the reverse, the driving of the ion pump by the hydrolysis motor, is also possible. The key power stroke for the F1 motor involves the binding of ATP to the catalytic site, whereas the exhaust stroke involves the hydrolysis of inorganic phosphate from ATP. In the FO motor, the flow of ions leads to the flashing of an electric field, which generates a torque to drive the motor. Oster said that all motors depend on the fundamental property of asymmetry. The catalytic state, $B$-sheet, and shaft are asymmetric in the F1 motor, which leads to counterclockwise rotation and sequencing. Similarly, the input and output channels are asymmetrically positioned in the FO motor.

M.E. McConney (Georgia Tech) described how some species of spiders use vibration sensing to detect prey. Wandering spiders (Cupiennius salei) found in Costa Rica and parts of South America do not build spider webs. Rather, they use a sit-and-prey technique to detect prey through vibrations. They use two different types of receptors: hair receptors for air-based vibrations and strain receptors for substrate vibrations through pads on their legs. The spider utilizes biopolymeric materials near their glass transition for mechanical signal filtering of vibration sensors. Atomic force spectroscopy was used to probe the time-dependent mechanical behavior of these materials and the Young's modulus of the rubberlike padshaped material situated between the stimulus source and the vibration sensors. Previous electrophysiological measurements showed a dramatic rise in the vibration amplitude needed to elicit an electrophysiological response below $10 \mathrm{~Hz}$ stimulation frequency. The elastic modulus measurements showed a similar decrease in stiffness as the frequency dropped to $\sim 10 \mathrm{~Hz}$, due to viscoelastic effects. The pad thus acts as a high-pass filter for the vibration sensors. In addition, point-load measurements on wind-sensing hair receptors demonstrated that the torsional stiffness of the hair suspension dropped off as the probing frequency decreased below $20 \mathrm{~Hz}$. The study showed that biology uses structural and mechanical properties for signal processing in addition to electrophysiological processing.

For further details on the research results reported at the 2008 MRS Spring Meeting, see the following symposium summaries. Proceedings as well as additional meeting highlights are available at the Web site www.mrs.org.

\section{TFT Applications of Amorphous and Polycrystalline Thin-Film Silicon Reviewed \\ (See MRS Proceedings Volume 1066)}

Silicon thin-film technology based on amorphous and polycrystalline silicon, the topic of Symposium A, dominates the world of large-area electronics, and through a combination of displays and solar cells, it supports an industrial market of many billions of dollars annually. This symposium, which has been held annually at the MRS Spring Meeting for 


\section{Government Funding Sessions Provide Overviews of U.S. Support for Materials Research}

Updated information regarding materials research funding opportunities available through the National Science Foundation (NSF), the Department of Energy (DOE), and the Department of Defense (DoD) was presented at government funding sessions held during the 2008 MRS Spring Meeting.

The overview of NSF opportunities emphasized two of the goals of the Division of Materials Research (DMR): broadening participation and international activities. One of the goals of DMR is to mentor young investigators to increase both the number of grant proposals they submit and the quality of the proposals. In this way, young investigators will be able to turn in proposals that are more competitive with those of established researchers. In 2008, DMR launched a pilot American Competitiveness Initiative (ACI) fellows program. DMR is also holding workshops in 2008 with the purpose of broadening the diversity of participants, including workshops on gender equity and scientists with disabilities. In regard to international collaborations, DMR has established numerous Materials World Networks with Central and South America and Western Europe, but little participation with Africa and Asia. This year, the division is pursuing further collaboration with China and Japan. Furthermore, new research-funding opportunities and directions within DMR include cyber-enabled innovation and discovery in materials research and education, transformative and innovative materials research, materials research at the interface between the physical and life sciences, biologically inspired materials, and green materials for the sustainability and advancement of humankind. The presentation was given by Zayka Kafafi, director of DMR.

At DOE, new directions in vision and challenges in specific areas have been identified based on a series of reports released since February 2003 and on a grand challenge subcommittee report released in January 2008. According to the latter report,

Air Force Office of Scientific Research (AFOSR) www.afosr.af.mil

Army Research Office (ARO) www.aro.army.mil

Department of Energy (DOE) www.science.doe.gov/grants

National Science Foundation (NSF) www.nsf.gov/materials

Office of Naval Research (ONR) www.onr.navy.mil the field needs to move from an "observational science" (20th century) to a "control and manipulation science" (21st century) in which the grand science challenges include the control of materials processes at the level of the quantum behavior of electrons, atom- and energy-efficient syntheses of new forms of matter with tailored properties, emergent properties from complex correlations of atomic and electronic constituents, artificial nanoscale objects with capabilities rivaling those of living things, and control of matter very far away from equilibrium. In FY2009, the DOE Office of Basic Energy Sciences proposes to establish a $\$ 100$ million Energy Frontier Research Centers (EFRCs) initiative. Under this initiative, universities, national laboratories, nonprofit organizations, and for-profit firms will be invited to compete, singly or in partnerships, to establish an EFRC. Centers will be selected by scientific peer review and funded at \$2-5 million per year over an initial 5 -year period. The purpose of these Centers will be to integrate the talents and expertise of leading scientists in a setting designed to accelerate research toward meeting U.S. critical energy challenges. The EFRCs will harness the most basic and advanced discovery research in a concerted effort to establish the scientific foundation for a fundamentally new U.S. energy economy. EFRCs will bring together critical masses of researchers to tackle fundamental energy research in a new era of science. Harriet Kung, director of the Division of Materials Science and Engineering, Basic Energy Sciences, at the Department of Energy presented the overview.

From DoD, Thomas Russell, director of Aerospace Chemical and Materials Sciences at the Air Force Office of Scientific Research (AFOSR), said that his department interfaces with universities in basic research to create scientific opportunities that the Air Force can use. His division is currently helping the mechanical community merge with the structural materials community, focusing on areas such as multiscale structural mechanics and prognosis, low-density materials, hightemperature aerospace, and mechanics of multifunctional materials and microsystems. Mihal Gross, a program officer in the Naval Materials Division of the Office of Naval Research (ONR), described research opportunities and needs for functional and structural materials, with current focus areas including high performance energy conversion and storage, propulsion, structural survivability, and environmental quality. The division supports long-range basic research as well as applied research and technology transition. David Stepp, chief of the Materials Science Division at the Army Research Office (ARO), said that his department wants research on unprecedented materials properties. ARO is looking for research 25 years out; that is, they want research that will have outcomes ready to implement for the soldier of 2033. the past 25 years, provides a unique forum for the discussion of both the fundamental materials science, physics, and chemistry that underpins current understanding of these materials and the means of production and technological challenges specific to given applications.

A particular focus of this year's symposium was thin-film transistor (TFT) applications, and a special session composed entirely of invited speakers offered a chance not only to review the progress made in this area over the past 30 years but also to consider the current state of the art. Meanwhile, in the area of solar cells, a key theme was the drive toward producing more efficient devices at ever lower cost over large areas, an area that undoubtedly will continue to grow rapidly as the need for energy from low-carbon sources increases. It was also noticeable that a number of novel applications of thin-film silicon technology are emerging, including MEMS devices, bolometers, and biomedical imaging devices, which serves to show how silicon continues to be a significant material for study.

Symposium Support: Asahi Glass Co.; AU Optronics Corp.; CYTEK Taiwan; Fuji Electric Advanced Technology Co.; Industrial Technology Research Inst.; Merck Chemicals UK; Trillion Science, Inc.; ULVAC Technologies, Inc.; and United Solar Ovonic LLC. 


\section{SiC Growth, Defects, and Devices Explored}

(See MRS Proceedings Volume 1069)

Silicon carbide $(\mathrm{SiC})$ is a very robust semiconductor material that is being actively developed for high-power and high-temperature applications, especially in the field of power electronics and sensors for harsh environments. Symposium $\mathrm{D}$ addressed $\mathrm{SiC}$ growth, defects, and devices. Among the highlights from the session devoted to bulk growth were the presentations by P. Wellmann (Univ. of Erlangen), who described efforts directed toward the growth of bulk $\mathrm{SiC}$. In addition, D. Hansen (Dow Corning Compound Semiconductor Solutions) discussed the influence of crystal growth conditions on nitrogen incorporation. Among the highlights of the sessions devoted to defects and characterization, P. Kaminski (Inst. of Electronic Materials Technology, Poland) discussed photoinduced transient spectroscopy of defect centers in undoped, semi-insulating 6H-SiC. A. Gali (Budapest Univ. of Technology and Economics, Hungary) described work on point defects in SiC, and Y. Chen (SUNY-Stony Brook) discussed synchrotron topography studies of dislocations in SiC. J.-P. Ayoub (Paul Cezanne Univ., Marseilles) reported on TEM studies of deliberately induced partial dislocations in $4 \mathrm{H}-\mathrm{SiC}$.

Among the highlights of the sessions devoted to epitaxial growth, M. O'Loughlin (Cree Inc.) described work on hot-wall epitaxy for large-area, high-voltage devices. H. Tsuchida (CRIEPI, Japan) discussed the influence of growth conditions on the formation of interfacial dislocations and half-loop arrays in 4H-SiC epitaxy. In addition, P. Bergman (Linköping Univ.; Norstel AB, Norrköping, Sweden) spoke about improved $\mathrm{SiC}$ epitaxial materials for bipolar applications. B. VanMil (NRL) discussed basal plane reduction using in situ growth interruptions. M.A. Capano (Purdue) presented information on graphene growth, and $\mathrm{H}$. Chen (SUNYStony Brook) addressed characterization and growth mechanism of $\mathrm{B}_{12} \mathrm{As}_{2}$ epitaxial layers grown on $6 \mathrm{H}$ and $15 \mathrm{R}-\mathrm{SiC}$.

In the session on deep-level defects and carrier lifetime, T. Kimoto (Kyoto Univ., Japan) reviewed deep levels and lifetimekilling defects in $4 \mathrm{H}-\mathrm{SiC}$ epilayers and J.A. Freitas (NRL) described the use of optical techniques for defect mapping in $\mathrm{SiC}$. In the sessions on devices and applications, J.D. Caldwell (NRL) discussed Shockley stacking fault propagation in $4 \mathrm{H}-$ SiC. P. Friedrichs (SiCED GmbH \& Co. KG, Germany) addressed considerations of bipolar power devices, and J.A. Cooper (Purdue) described the design and fabrica- tion of high-voltage, $n$-channel DMOS IGBTs on $4 \mathrm{H}-\mathrm{SiC}$ free-standing epilayers. P.G. Neudeck (NASA Glenn Research Center) discussed the long-term characterization of $6 \mathrm{H}-\mathrm{SiC}$ transistor IC technology operating at $500^{\circ} \mathrm{C}$. In addition, L. Tolbert (ORNL; Univ. of Tennessee) described SiCbased power converters. Among the highlights of the session on MOS interfaces, J.R. Williams (Auburn Univ.) addressed $\mathrm{SiO}_{2}$ and $4 \mathrm{H}-\mathrm{SiC}$ interface issues.

Symposium Support: Cree, Inc. and Sula Technologies.

\section{Device Technologies with SiC Materials Rapidly Maturing}

\section{(See MRS Proceedings Volume 1070)}

Symposium E focused on doping engineering for front-end processing. The doping optimization of source and drain regions, including reduction of contact resistance between silicide and $\mathrm{Si}$ in MOS devices, is a trade-off between short channel effect control, drive current, and junction leakage. High activation, good control of dopant profile, and perfect $\mathrm{Si}$ quality without any residual defects are the key elements to improving device operation. Implementation of stress techniques in device channels enhances carrier mobility and therefore very efficiently improves drive.

Preamorphization followed by solidphase epitaxial regrowth (SPER) is generally used to achieve good dopant activation with negligible dopant migration. Although the SPER process is applied at low temperatures, some dopant diffusion occurs during the amorphous Si phase, which affects the final dopant profile. This is true for all activation methods that require amorphization of $\mathrm{Si}$ prior to $\mathrm{B}$ implantation. S. Mirabella (MATIS, CNRINFM, Catania, Italy) characterized B diffusion in amorphous Si by using MBE-grown B markers in SOI wafers. Mirabella's research group showed that $\mathrm{B}$ diffusion has a transient character and strongly depends on concentration. They proposed that B diffusion is enhanced by the presence of dangling bonds, whose formation is favored by the presence of B itself.

The Si recrystallization kinetics of implantation-induced amorphous layers is affected by crystal orientation and stress. Using TEM images, K.S. Jones (Univ. of Florida) examined the 2D shape of the amorphous/crystalline interface as well as the defect formation process in a bending wafer and patterned thin films. In planar interfaces, both compression and tension resulted in an increase in regrowth defects compared to the stressfree case. In patterned wafers, the stress effects on SPER velocity for different crystallographic orientations can prevent extended defect formation at mask edges.

Amorphization in ultrathin-body devices poses a severe problem because of slow $\mathrm{Si}$ regrowth along $\mathrm{Si} / \mathrm{SiO}_{2}$ interfaces and insufficient material quality. M. van Dal (NXP Semiconductors) provided an overview of challenges in the fabrication of low-resistive source/drain extensions in aggressively scaled $\mathrm{Si}$ devices, such as multigate FinFETs. The difficulties in dopant retention and thin $\mathrm{Si}$ body recrystallization in narrow fins were identified as being responsible for the degradation of device performance.

N. Breil (STMicroelectronics, France) demonstrated an important Schottky barrier height $(\mathrm{SBH})$ modulation for As and B segregation by using an implant before silicidation. He showed that the activation of As before the silicidation does not impact the SBH modulation but that the dopant crystalline position has a significant impact for boron. By comparing conventional implant versus a plasma doping, he showed the suitability of the latter implantation tool for SBH modulation.

New Materials, Integration, and Device Architectures Discussed for Advancement of Nonvolatile Memories (See MRS Proceedings Volume 1071)

Symposium $F$ focused on the science and technology critical to development of the next generation of nonvolatile memories (NVMs). Emphasis was placed on discussing the development of new materials, materials integration, and device architectures scaling to nanoscale dimensions to expand the scalability of current mainstream "Flash" memory technology, and enable new types of nonvolatile memories that are promising alternatives for next generations of nanoscale-NVMs.

Invited talks focused on the research of new materials for Flash memory scaling, including the development of new high- $k$ dielectric stacks for improved gate coupling (without compromising retention), and the search for new materials for charge trap memories (that may replace silicon nitride). Several talks focused on the description of alternative memory concepts that are no longer based on charge storage but rather on other mechanisms such as programmable resistance (resistive switching memories). Discussions in this space were marked by the departure of the basic device from dependence on $\mathrm{Si}$ as the base material; instead, other functional materials were proposed such as metal oxides; ferroelectric, magnetic, and ferroic materials; chalcogenides (e.g., as phase-change materials); organic materials (including polymers); and on 
new nanomaterials and nanostructures like carbon nanotubes, and single-crystal nanowires. The decoupling of the device from the Si substrate enables integration strategies whereby the memory elements move up into the interconnect network and can be, for example, integrated into contact or via structures of 3D memory arrays. In some cases, these functional materials are also combined with nanomechanical structures in new nanoprobing concepts.

C. Araujo (Symetrix Corp.; Univ. of Colorado) reviewed the status of nonvolatile FeRAMs and the mass-market production for products such as "smart cards," RFID devices, and other applications expected to come in the future. This talk revealed that after many years of R\&D, the FeRAM technology has matured for the low-density memory range, and the next frontier is R\&D to enable highdensity ( $\mathrm{Gb}$ and $\mathrm{Tb}$ ) FeRAMs.

Symposium F was aimed at providing a forum to stimulate new ideas toward fundamental and applied materials research as well as device design and fabrication of nanostructures, which will be necessary to understand the nanoscale structure-property relationships of the thin films and their novel hybrid combinations planned for use in the fabrication of future NVMs.

Symposium Support: Applied Materials, Inc.; Numonyx; Seagate Technology; and Symetrix Corp., Colorado Springs.

\section{Ceramic and CMOS-Compatible Materials Studied in Passive and Electromechanical Materials and Integration \\ (See MRS Proceedings Volume 1075E)}

Two areas for materials research in Symposium J drew particular attention. The first considered ceramic materials:

- materials with a high dielectric constant (high $\kappa$ ) and low loss, such as PZT, BST, STO, CCTO, and $\mathrm{LaNiO}_{3}$ (A.I. Kingon, NCSU; Y. Cho, Yonsei Univ.; S. Trolier-McKinstry, PSU; B. Tuttle, SNL; T. Tsurumi, Tokyo Inst. of Technology);

- materials with voltage-controlled permittivity, such as BST and STO (T. Suzuki, Taiyo Yuden Co.; M. Al Ahmad, LAAS); and

- piezoelectric materials, such as PZT (P. Muralt, EPFL; C. Ayela, LAAS; E. Fujii, Matsushita).

This research is triggered by the requirements of future handheld multiband radios capable of operating in multiple cellular standards like GSM, GPS, DVB, and WiMax. Future miniaturization of the radio front end is partly achieved by integration and reconfigurability of passives. High-density capacitors implemented with high- $\kappa$ thick- or thin-film dielectrics can be integrated in the substrate, leading to an rf-SoP (N.K. Kang, Korea Electronics Technology Inst.). Reconfigurable rf components can be made by using voltage-tunable ceramics or by implementing piezoelectric MEMS capacitors and switches.

The second area addressed more CMOS-compatible materials:

- SiGe (A. Witvrouw, IMEC) and

- nanowires and nanotubes (P.X.L. Feng, Caltech; S. Park, Gyeongsang Natl. Univ.).

The SiGe thin film is considered a versatile material for MEMS, particularly gyroscopes and mechanical resonators. Apart from its excellent mechanical properties, an attractive feature of $\mathrm{SiGe}$ is its low deposition temperature $\left(<42^{\circ} \mathrm{C}\right)$, which allows above-CMOS integration. Nanotubes and nanowires are emerging as base structures for ultrasensitive resonant-mode detections of mass, force, and charge; as novel gas sensors; and as rf signal-processing elements. CNT scaffolding is being investigated as a novel method for producing high-aspect-ratio MEMS applicable for a variety of materials (D.N. Hutchison, Brigham Young Univ.).

Symposium Support: IBM Almaden Research Center and MRS Nano Instruments.

\section{Materials Progress in Laser Remote Sensing and Optical Communication Reported}

(See MRS Proceedings Volume 1076)

Laser remote sensing and optical communication are two important technology areas with great potential to improve human lives and impact the global economy. Although these two fields are distinct in their application areas and marketplaces, they share many common technology elements such as lasers, detectors, modulators, and other photonic and semiconductor devices. Laser remote sensing finds major applications in areas as diverse as atmospheric studies and climate research, marine ecology, weather forecasting, space studies, air travel safety and efficiency, and military target identification and countermeasure, to name a few, thus addressing significant societal needs. Among the goals of Symposium K were recognizing the cross-cutting technologies serving both of these fields, improving the understanding of laser remote sensing needs that can be addressed by the optical communication researchers and materials scientists, and familiarizing the remote sensing community with the advanced component technologies offered by optical communication technologists. The tutorial on pulsed fiber lasers provided an appropriate start for this symposium by discussing the recent advances in pushing the operational envelope of pulsed fiber lasers and discussing the potential and limitations of pulsed fiber lasers for remote sensing applications. In addition to serving as the instructor of the tutorial, M. Fermann (IMRA America, Inc.) presented a talk on high-power ultrafast fiber lasers, which are of importance to several laser remote sensing applications. A thorough assessment of fiber lasers capabilities and their potential for optical communication and remote sensing applications was provided by P. Russell (Univ. of Erlangen, Germany).

Any improvement in the efficiency of detection devices will directly translate into lower required transmitter laser power, resulting in more compact and efficient LIDAR instruments. A number of novel detector technologies were presented. M. Shur (RPI) reported on the most recent advances in plasma wave detectors for terahertz sensing technology, which has applications in the detection of biological and chemical hazardous agents and explosives, as well as potential applications in medicine such as in cancer detection. Focal plane array devices based on the avalanche photodiode technique are attracting a great deal of attention for both active and passive remote sensing and imaging such as identification of military targets and 3D mapping of terrain. M. Reine (BAE Systems) reviewed the physics and characteristics of electron-initiated $\mathrm{HgCdTe}$ avalanche photodiodes ( $\mathrm{HgCdTe}$ e-APDs) and reported on the current state of the technology.

Quantum cascade lasers are an emerging technology in providing highly coherent radiation in the mid-infrared range. M. Razeghi (Northwestern) reported the development of a novel quantum cascade laser utilizing a 2D photonic crystal distributed feedback (PCDFB). The PCDFB technique can greatly enhance the coherence of the laser beam, thus making single-mode operation possible for broad-area devices. F. Capasso (Harvard) discussed broadband quantum cascade laser-based spectrometers for chemical and biological sensing, as well as quantum cascade plasmonic laser antennas for subwavelength-resolution chemical imaging.

Symposium Support: Coherent Applications; Discovery Semiconductors, Inc.; IPG Photonics Corp.; NASA Langley Research Center; NSF; and PolarOnyx, Inc. 


\section{Sensors and Transistors Stretching to New Heights \\ (See MRS Proceedings Volume 1078E)}

Electronic devices of the future will be able to stretch, fold, and twist in ways that create many new application opportunities that lie beyond the capabilities of conventional systems built on the rigid, brittle surfaces of glass or semiconductor wafers. Symposium $M$ highlighted recent advances in the materials, mechanics, and applications in this emerging area of technology. As examples of the former, T. Sekitani (Univ. of Tokyo) and C. Liu (Northwestern), Q. Cao (UIUC), and Y. Sun (ANL) showed how CNT composites and networks could be used for conducting, semiconducting, and sensing elements, respectively, of flexible and stretchable circuit and sensor systems. Y. Huang (Northwestern) and Z. Suo (Harvard) presented detailed analytical and finite element models for the mechanics of these and other systems, thereby providing clear design rules and optimized layout possibilities for achieving desired mechanical properties. S. Wagner (Princeton Univ.) highlighted some of the challenges associated with demonstrating new applications with emerging technologies, especially for multilayered structures on stretchable substrates.

On the application side, J.S. Gregory (Conemaugh Memorial Medical Center; UIUC) presented some interesting concepts involving circuits and actuators in rubber surgical gloves designed for a virtual operating theater environment. Prototype devices, including some with balloon-type haptic actuators, demonstrated some of the ideas and also highlighted remaining materials challenges. S. Bauer (JohannesKepler Univ.) described novel polymeric electret materials that could form sensing skins capable of localizing a stimulus in three dimensions. J. Vanflateren (TFGC Microsystems, Belgium) described multiple stretchable electronic circuit applications capable of functioning under conditions of $100 \%$ stretch.

Symposium Support: ALA Scientific Instruments, Inc.; Multi Channel Systems; Nokia Corp. and Plexon, Inc.

\section{Low-k Dielectrics Highlighted for Advanced Interconnects for Microelectronics}

\section{(See MRS Proceedings Volume 1079E)}

The semiconductor industry is moving into the 32-nm node with minimum onchip wire dimensions of $50 \mathrm{~nm}$ or less. Aggressively scaled interconnects require continued innovations for providing fast signal propagation, low power consumption, and adequate reliability. In addition to evolutionary approaches related to further dimensional and material scaling, several alternatives such as 3D interconnects, 2D and 3D self-assembly, and nanotube-based interconnects are being actively researched.

Many presentations in Symposium N related to the processing of low $k$ dielectrics. Plasma interactions, the impact of UV treatments, mechanical strength, and $k$-value restoration treatments were well represented at the symposium. Approaches related to $k$-value scaling of dielectric cap barrier layers in efforts to further reduce the integrated $k$ value were discussed in several presentations.

It remains challenging to find low- $k$ dielectrics with $k$ values below 2.5 that have adequate mechanical properties and adequate stability to plasma modifications and that can be patterned at small dimensions. Apart from a few exceptions, most of the presentations on the topic of low $-k$ dielectrics focused on materials with a dielectric constant of 2.5. For example, Y. Hayashi (NEC) explained how molecular-level manipulation can enable 32-nm integration with an integrated $k$ value of 2.75. Other presentations on novel deposition methods and patterning methods included the deposition of low- $k$ materials by ALD and the patterning of low- $k$ materials by nanoimprint lithography.

The sessions focused on metallization provided a varied overview of the current status and recent advances. As the scaling of traditional Ta-based metallization is becoming increasingly challenging, a large variety of potential alternatives are being considered. For example, there were reports on novel barrier and seed layers, using $\mathrm{Co}_{4} \mathrm{~N}, \mathrm{CVD}$ of $\mathrm{Cu}$, SAMs, or Mn-based self-forming barriers. Ru-based barriers, and related metals such as Ir and Os, were also discussed. In particular, selfforming Mn-based barriers are attracting significant attention. R.G. Gordon (Harvard) explained how these barriers can be deposited using an ALD-based approach, while J. Kioke and co-workers from Tohoku University detailed the impact of low $k$ surface modifications on $\mathrm{Mn}$ reactions at the $\mathrm{Cu} /$ low $-k$ interface. Plating of copper, grain growth, and the relation between grain growth and the resistivity of copper were also detailed.

A morning session was focused on reliability and packaging aspects. C. Hau-Riege (AMD) explained how short line effects and porous barriers can impact electromigration performance. Some aspects of mechanical and dielectric reliability were explained in other contributions. Chip stacking and lead-free solders were also discussed.
The emerging interconnect session was focused mainly on CNT growth, characterization, and integration. Currently, nanotubes are being considered for via and interconnect applications. C.V. Thompson (MIT) argued that using CNTs in highaspect-ratio vias would enable significantly reduced power consumption. There was also a demonstration of a ring oscillator with a carbon nanotube interconnect by G. Close (Inst. for Materials Science, Leoben, Austria).

The need continues for further improvements in on-chip interconnects.

\section{Semiconductor Nanowires Featured for Energy Conversion and Storage (See MRS Proceedings Volume 1080E)}

The field of semiconductor nanowires is extremely active and has progressed tremendously in recent years. This was reflected not only in the contributions in the area of advanced growth and improved characterization of materials, but also in the large number of different applications demonstrated. Applications for semiconductor nanowires presented at Symposium O included FETs, LEDs, memory cells, sensors, and solar cells. The maturity of nanowires was also highlighted by the electrical performance demonstrated in nanowire FETs, which are now comparable to state-of-the-art CMOS devices.

Of particular interest is the use of nanowires for energy conversion and storage, such as solar cells and batteries as addressed by Y. Cui (Stanford) in his invited lecture. Many unique properties, including large surface area and nanoscale diameters combined with facile strain relaxation and charge-transport capability, make nanowires ideal materials for energy storage. Using novel Li-ion battery electrodes based on Si nanowires, Cui was able to demonstrate their ultrahigh capacity as compared with existing technology.

Another exciting discovery is that silicon nanowires can form excellent thermoelectric materials, which might enable new applications in power generation, refrigeration, and heating. Both $\mathrm{A}$. Boukai (Caltech) and A.I. Hochbaum (UCBerkeley) demonstrated that nanostructuring significantly increases the efficiency of $\mathrm{Si}$ as a thermoelectric material. ZT values of up to 1 were measured in Si nanowires, which is a 100 -fold improvement over bulk $\mathrm{Si}$ and renders them closer to commercial thermoelectrics. The dramatic increase of ZT is due to phonon effects that arise at the nanoscale and favorably affect the thermal conductivity and thermopower. Further research needs to be done to fully understand the physics 


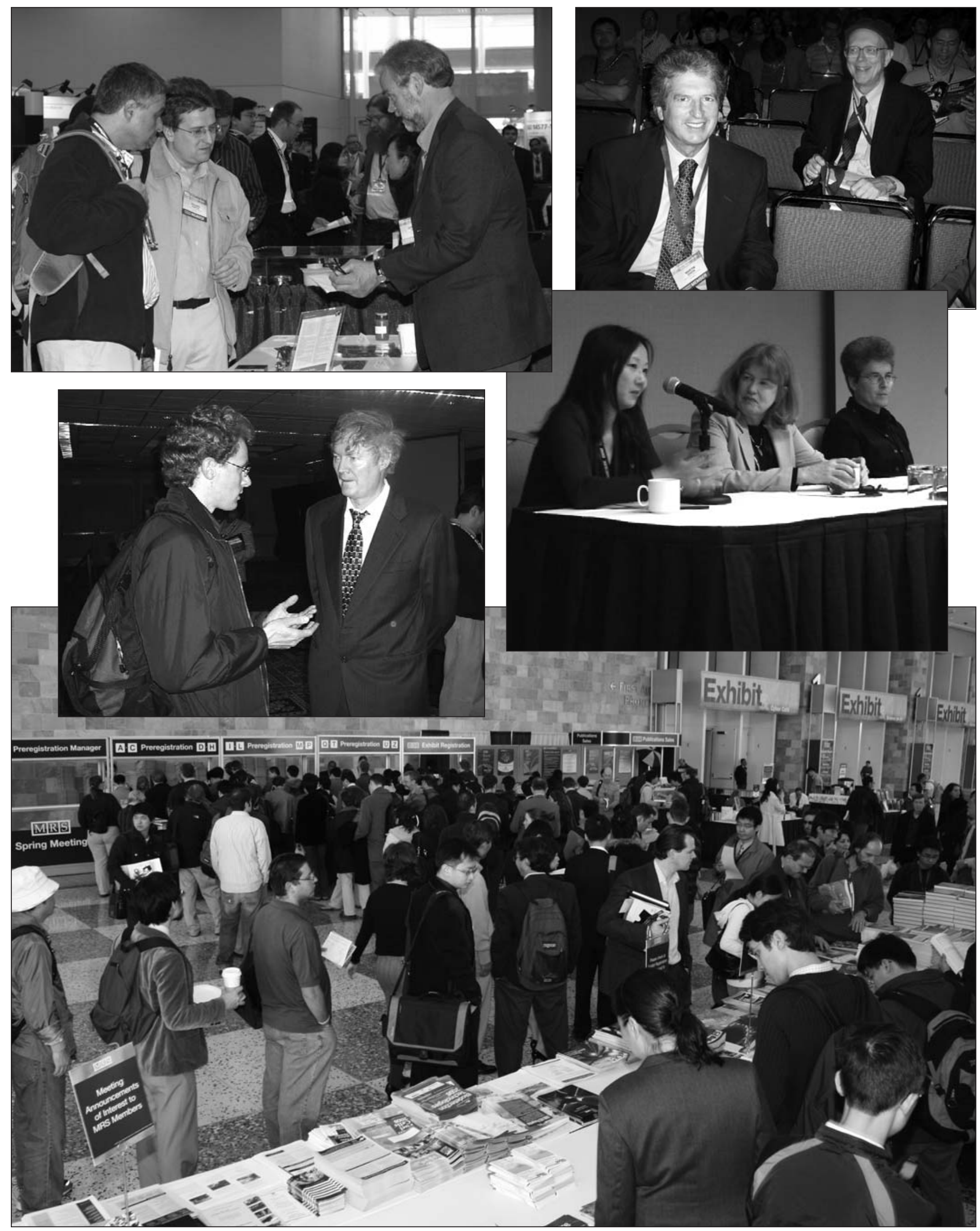




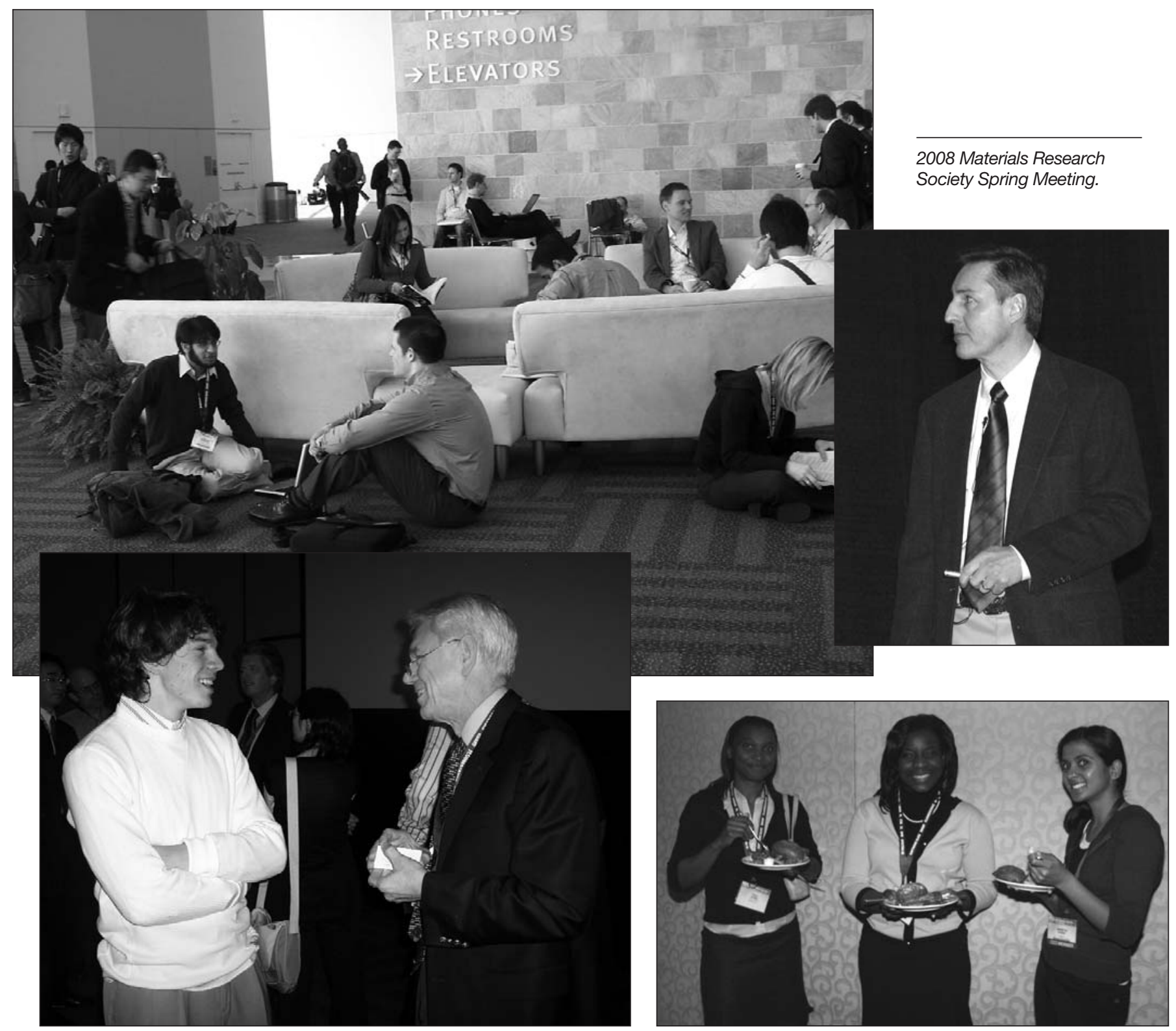

behind this effect and employ nanowires for thermoelectric applications.

Symposium Support: Cambridge Nanotech, Inc.; FEI; IBM Research Zurich; Oxford Instruments Plasma Technology; Raith USA, Inc.; and Veeco Instruments, Inc.

\section{Progress in Graphene CNTs Reported} (See MRS Proceedings Volume 1081E)

In Symposium $\mathrm{P}$, graphene attracted much attention because its unusual band structure corresponds to a linear dispersion through the Fermi point, giving charge carriers in graphene unusual symmetry properties and extremely large mobilities. The quantum Hall effect has been observed in graphene. W.A. de
Heer (Georgia Tech) summarized work on growing epitaxial graphene layers on the hexagonal faces of a SiC substrate by heating and the electrical data measured for these layers. He contrasted the graphene made from $\mathrm{SiC}$ with that made by the Geim method of exfoliating graphite using Scotch tape. According to de Heer, a few-layer system of this graphene can still act like a single layer because of a complicated rotational distortion as reported recently by J. Hass (Georgia Tech) and colleagues in Physical Review Letters (2008; DOI: 10.1103/ PhysRevLett.100.125504).

P. Kim (Columbia Univ.) compared carrier mobilities and scattering lengths in graphene to those observed in GaAs HEMTs as a function of temperature. Kim uses the Scotch tape method. He recently attained a maximum mobility of $200,000 \mathrm{~cm}^{2} / \mathrm{Vs}$ by passing a current through the graphene sheet to drive off impurities. Interestingly, this is still two orders of magnitude below the peak mobility in HEMTs. He also showed how cutting the graphene layer into strips opens a bandgap. De Heer reported that he was still achieving high mobilities despite the atomically rough edges.

De Heer said that the $\mathrm{SiC}$ method originated 20 years ago and has required considerable development by his group at Georgia Institute of Technology. Never- 
theless, P. Ye (Purdue) and colleagues are also growing graphene from $\mathrm{SiC}$.

R.S. Ruoff (UT, Austin) exfoliated graphite into single layers, the graphene oxide route or Hummer method. This can form a suspension. The graphene oxide can be reduced back to graphene by hydrazine, and the graphene sheets can form a low-concentration percolating conductive network, just like nanotubes.

Regarding nanotubes, talks on growth were presented by D.E. Resasco (Univ. of Oklahoma) and G. Eres (ORNL). Resasco described growth of SWNTs using the designer catalyst CoMoCAT, which was specially designed to work with $\mathrm{CO}$ as the carbon source, rather than the usual hydrocarbon. It gives small-diameter SWNTs, as the $\mathrm{CO}$ oxidizes the molybdenum oxide, expelling the Co, which then sits on the surface as Co clusters, as described in articles in the Journal of the American Chemical Society (2003; DOI: 10.1021/ja036622c) and the Journal of Catalysis (2004; DOI: 10.1016/ j.jcat.2003.08.005). Eres described the ORNL work on understanding the growth process and growth kinetics of CNTs, using measurements of growth rates as a function of temperature and pressure. One objective is to understand what causes catalyst poisoning

P.M. Ajayan (Rice) summarized his work over many years on growing nanotubes for novel applications. Various organized architectures of multiwalled and single-walled carbon nanotubes can be fabricated using CVD techniques. A few methods for attaining control of the directed assembly of nanostructures on various templates were highlighted.

K.-H. Chen (Academia Sinica, Taiwan; Natl. Taiwan Univ.) presented a direct growth approach of dispersing $\mathrm{Pt} / \mathrm{Ru}$ and $\mathrm{RuO}_{2}$ nanoparticles onto wafer-scale arrayed CNTs. Such a microdevice platform for electrochemical energy applications, including fuel cells and supercapacitors, has shown superior efficiency in current collection and charging-discharging properties using only one-tenth of the precious metal. A related talk on the electrochemical aspects of carbon flakes, or multiwalled graphene, was presented by P. Papakonstantinou (Univ. of Ulster, UK). She showed that the inherently large amount of graphitic edge planes in this material exhibited extremely high surface activities, making carbon flakes

\section{Nanoscience and Engineering Incorporated into Education}

Presentations in Symposium OO on the Role of Lifelong Education in Nanoscience and Engineering concentrated for the most part on successful programs conducted in classes and workshops from $\mathrm{K}-12$ to university to public outreach venues. Marie-Isabelle Baraton of the University of Limoges, France, stepped outside the "how to" realm in order to describe the European Union's significant investment in nanoscience and nanotechnology (N\&N), including special plans for education. With $€ 4,200$ million budgeted for $N \& N$, the European Commission (EC) is the largest investor worldwide in this area of science. The EC wants to enable "knowledge transfer" across the continent in N\&N. According to the commission, a knowledge-based society requires interactions between research, education, and innovation. Some European universities are concentrating on designing postgraduate degrees that provide scientists and engineers with the multidisciplinary skills necessary for work in N\&N; some of these programs include elements of entrepreneurship or business skills. New nanoscience and technology courses are prominent in Germany and the United Kingdom; and Denmark has developed short courses in this field. The European Union is also working toward mutual recognition of degrees in order to allow mobility across countries in work and study. Education and training are key to eliminating barriers to nanotechnology commercialization. Toward this end, universities across Europe are engaged in a process of modernization stimulated by the Bologna Process that aims to create a European Higher Education Area (EHEA) by 2010.

In the area of public outreach, Alex Prasertchoung and his advisor, Donna Hammer, presented a program to motivate the next generation of crime scene investigators through the newest exhibit at the Spy Museum in Washington, DC, "Mission Possible, the Art of Spying." In activities highlighting science behind the scenes, participants identify targets using sensors, imaging, codes, and mission microdots. Museum outreach efforts included "Operation Night Spy" and "Teacher Spy Science Workshop." Carl Batt described two exhibits developed with support from the National Science Foundation through the Cornell Nanoscale Science and Technology Facility: "It's a Nano World" and "Too Small to See." The key focus of the Center for middle school motiva-

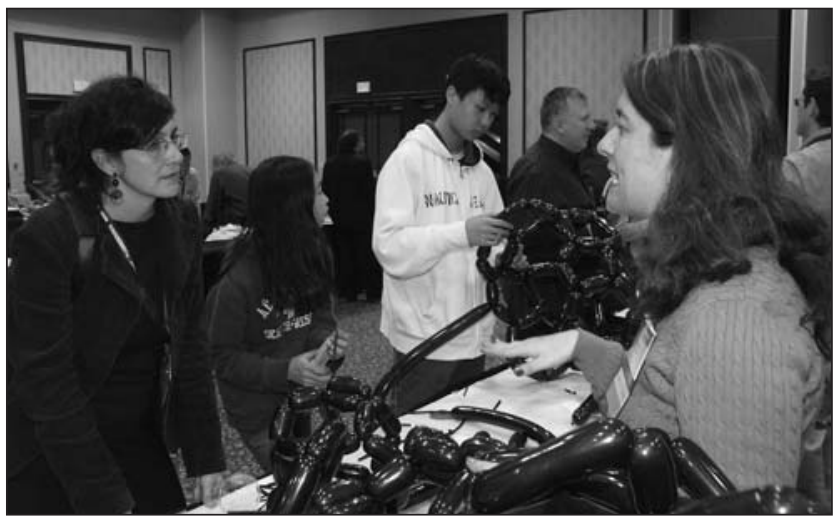

Attendees were invited to join with national science centers, universities, and educational research centers for an evening of fun with science and engineering at the Education Outreach showcase.

tion in nanoscience is the online magazine called Nanooze (www.nanooze.org).

Kim Duncan said that the University of WisconsinMadison's approach to outreach involves reaching out to the general public and professional community to improve understanding of nanotechnology and to develop nanoresearch responsibly. In a workshop promoted through the school of public policy, participants are encouraged to engage in handson laboratory activities, take laboratory tours, and receive input from nanotechnology researchers on current nano efforts. In this process, nano-misconceptions can be dispelled, and more responsible nanotechnology public policy can be generated, said Duncan.

Presentations will be published in MRS Proceedings Volume 1105E. The symposium received support from Asylum Research Corp.; CYTEC Industries, Inc.; FEI Co.; Georgia Tech, Microelectronics Research Ctr.; Hitachi High Technologies America; JEOL USA, Inc.; NNIN; Small Times Magazine/Penn Well Corp.; SPECS Technologies Corp.; Super Conductor Materials, Inc.; Thermo Scientific; and Veeco Instruments, Inc. 
a promising option for use in catalytic electrodes and biosensors. M. Meyyappan (NASA, Ames Lab.) has a similar outlook. He summarized work on nanotubes for chemical sensors, beginning with a specification of sensor requirements, particularly in the area of security. A closely related talk on nanotubes for sensors was presented by E. Snow (NRL), who showed the two sensing methods, capacitive and resistive. Introducing defects such as vacancies creates binding sites that increase the sensitivity and can also be used to give a large dynamic range. However, too many defects can affect resistivity, so this technique should not be overdone. Sensitivities of down to 1 part in $10^{13}$ were possible for some gases, some orders of magnitude better than those obtained by other groups.

J.A. Rogers (UIUC) described work on nanotube TFTs. The standard nanotube TFT consists of "bucky paper" with a randomly oriented mat of tubes. Given that this material contains both semiconducting and metallic nanotubes, care must be used with this format to tune the nanotube density so that good values of the on/off ratio and on current are obtained simultaneously. Recently, it has been found that certain surfaces of sapphire and quartz will cause nanotubes to grow with parallel alignment, in a flat layer over the surface. Now, it is possible to lithographically cut strips of nanotubes mat along their orientation and make much better transistors. M. Chhowalla (Rutgers), in presenting related work, summarized his recent studies on conducting nanotube mats and percolation behavior. He then described similar work using graphene sheets instead of nanotubes, with the graphene prepared by the Ruoff method. In principle, this graphene route actually looks less expensive and more scalable than those used to obtain the nanotube material, given that only simple wet chemistry and filtering are involved.

M.C. Hersam (Northwestern) described his research on the separation of SWNTs by chirality. An interesting but problematic feature of SWNTs is that they consist of a mixture of nanotubes with many chiralities of very similar free energies that can be both metallic and semiconducting. Three years ago, three separation methods were available. Hersam applied the DNA method developed by DuPont, which tends to separate according to diameter. He explained how to use surfactants, ultracentrifugation, and density gradients to separate the nanotubes according to their solvated density. Initially, this gives separation by diameter and can be repeated until it gives separation by chirality. Infrared absorption and photolumines- cence spectroscopies show the degree of separation. The resulting material is then of great interest to those groups making transistors or optical devices.

J.E. Fischer (Univ. of Pennsylvania) described his studies of the thermal conductivity of nanotubes and nanotube composites. Nanotubes, in principle, have an axial thermal conductivity of similar order to that of diamond. They can also be added to polymers to make composites. However, as summarized by Fischer, improving the thermal conductivity of these composites has been a long, tough job.

C. Zhou (Univ. of Southern California) produced nanotube FETs. Nanotubes have very high mobilities and exhibit ballistic transport over short distances, so in principle, they have very high figures of merit as transistors, as shown some time ago by a group at IBM. However, Zhou described the difficulties encountered in making the transistors; for example, it is necessary to carefully dope the nanotubes and contacts to prevent them from having ambipolar conduction, which causes them to turn off.

Z. Liu (Peking Univ.) described work on the engineering of nanotubes for devices. For example, a typical study consisted of varying the growth temperature along a nanotube, so that its diameter and thus bandgap varied along its length. Various other forms of control were also demonstrated.

Symposium Support: Aixtron and Natl. Taiwan Univ.

\section{Ionic Liquid Materials Offer New Research Directions}

(See MRS Proceedings Volume 1082E)

Ionic liquids have been used as thermally stable and low-volatility/nonvolatile solvents in a broad range of applications, from processing of cellulosic materials to construction of dye-sensitized solar cells. Symposium $\mathrm{Q}$ addressed ionic liquids in materials synthesis and applications. R.D. Rogers (Queen's Univ. and Univ. of Alabama) discussed state-of-the-art approaches using ionic liquids in the processing of biomaterials. He said that ionic liquid materials and biological ionic liquids could be new research directions and emphasized the needs for green-chemistry-guided research in the future. T. Lodge (Univ. of Minnesota) presented work on functional ion gels obtained by self-assembly of block copolymers in ionic liquids and their potential in device fabrications. The use of ionic liquids in studies of biological processes such as protein folding and dissolution and enzymatic transformation of carbohydrates was addressed by
F.V. Bright (SUNY-Buffalo), C.A. Angell (ASU), and H. Zhao (Savannah State Univ.). Electronic applications of ionic liquids were presented by T.M. McCleskey (LANL). E.W. Castner (State Univ. of New Jersey), M. Shiflett (DuPont), and M. Maroncelli (PSU) presented the fundamental aspects of ionic liquids related to materials processing, such as solvation.

An emerging area of ionic liquids in materials applications is their use in the synthesis of nanostructured materials. P. Alivisatos and P.D. Yang, both of the University of California-Berkeley, and Y.N. Xia of Washington University presented mechanistic studies of colloidal nanocrystals with controlled shapes and compositions in molecular solvents. Alivisatos said that ionic liquids might also play a role in mechanistic studies of II-VI semiconductor nanocrystals. J. Dupont (UFRGS) described the unique "cage" structures of ionic liquids in the synthesis of nanoparticles in ionic liquids, and S. Dai (ORNL) discussed the syntheses of novel ionic liquids for sol-gel and low-dimensional materials.

Symposium Support: AFOSR.

\section{Nanomaterials Offer Solutions in Coupled Behavior Problems} (See MRS Proceedings Volume 1083E)

Symposium R on coupled mechanical, electrical, and thermal behaviors of nanomaterials brought together noted researchers in multiphysics modeling, simulations, and experiments on nanomaterials and nanostructured materials. Invited lectures focused on a range of issues for using nanomaterials in situations where coupled behavior is an important problem. P.M. Ajayan (Rice), Y. Huang (Northwestern), and J.W. Kysar (Columbia Univ.) discussed carbon nanotube modeling and applications. Z.L. Wang (Georgia Tech) described work on zinc oxide nanobeltbased piezoelectric devices. H. Gao (Brown) focused on the modeling of biological cell adhesion, whereas N.R. Aluru (UIUC) and $\mathrm{K}$. Bhattacharya (Caltech) described the multiphysics and multiscale modeling of nanoelectromechanical systems. G. Chen (MIT) and A. Majumdar (UC-Berkeley; LBNL) discussed thermal and electrical transport in inorganic and organic nanostructured materials. H.T. Johnson (UIUC) and J. Qu (Georgia Tech) described the modeling of defects in electrochemical systems. I. Baker (Dartmouth College) discussed work on the development of magnetic nanoparticles for hypothermia treatment, and S. Kaskel (Technical Univ. Dresden, Germany) addressed functional polymer design. Each of these invited lectures provided 
information on a range of outstanding issues that need to be solved for significant developments in the current state of the art in the science and technology of coupled behavior. Contributed talks well complemented the invited lectures with focuses ranging from nanoscale piezoelectric behavior and nanoscale thermometry to multiscale biological chemomechanical modeling.

Symposium Support: AFOSR.

\section{Weak Interaction Phenomena Explored through Modeling and Simulation from First Principles} (See MRS Proceedings Volume 1084E)

Interest in weakly bound systems has surged recently because of a range of new functional materials and novel applications. Energy-related research also necessitates a better understanding of weak interactions. For example, the ideal hydrogen storage medium would be a porous but otherwise solid-state host to which hydrogen molecules bind through a weak interaction. Symposium S, Weak Interaction Phenomena-Modeling and Simulation from First Principles, fostered activities in the development of first-principles methods to model and simulate weak binding interactions in realistic materials. D.C. Langreth (Rutgers) and M.R. Pederson (NRL) addressed the importance of van der Waals interactions and presented the results of recent density functional theory studies on van der Waals interactions. In addition, M. Cote (Univ. of Montreal) explained another recent successful development of density functional theory in timedependent electron-density systems with application to polymers.

On the other hand, G. Galli (UC-Davis) K. Umemoto (Univ. of Minnesota), and X.C. Zeng (Univ. of Nebraska-Lincoln) described rich structural properties of water molecules in various environments where weak interactions also play an important role. In addition, Y. Kanai (UC-Berkeley), Y. Morikawa (Osaka Univ., Japan), and M. Rohlfing (Univ. of Osnabrueck, Germany) showed results on the interactions of molecules on various surface systems where weak interactions can play an essential role.

Symposium Support: LLNL.

\section{Mechanics of Nanoscale Materials Explored}

(See MRS Proceedings Volume 1086E)

Wide ranges of materials, techniques, and phenomena associated with the mechanical behavior of nanoscale materials were discussed during Symposium U. Many biological materials can be viewed as sophisticated composites whose unique combination of mechanical properties derives from an architectural design that spans nanoscale to macroscopic dimensions. R.O. Ritchie (UCBerkeley; LBNL) presented attempts to develop a range of biologically inspired bone-like and nacre-like structural materials using a new freeze-casting technique. This approach utilizes the intricate structure of ice to create hybrid materials with complex lamellar and/or mortarand-brick structures modeled across several length-scales that lead to novel and enhanced fracture properties.

Several presentations emphasized the importance of the defect structure for the mechanical behavior of crystalline nanoscale materials. K. Sieradzki (ASU) argued that, in small-volume systems, there can be a probabilistic aspect to the distribution of defects, resulting in a statistical nature to the deformation behavior. He applied these ideas to the case of nanoscale pillars and showed how such statistical considerations can be used to understand the compression behavior. H. Van SwygenhovenMoens (PSI) presented work using an in situ microcompression device allowing the continuous measurement of time-resolved white-beam Laue diffraction patterns during compression of pillars that captured the initial microstructure and the changes in microstructure during deformation. Her results demonstrated that evolution of the defect structure that occurs during compression can critically influence the strength of these materials.

Sandwiched between the domains of electronics and photonics, the terahertz regime of the electromagnetic spectrum has traditionally lacked mature technologies for generation, mixing, and detection. Yet, the terahertz regime has important applications, from the detection of 
chemical and biological agents to imaging and telecommunications uses. Addressing this gap, J. Reno (SNL) presented results from his group in developing terahertz quantum cascade lasers during Symposium B on Progress in Semiconductor Materials IV: Electronic and Optoelectronic Applications. Using a resonant tunneling longitudinal optical-phonon scattering technique for lower-level depopulation and a novel double-sided metal-metal waveguide for transverse confinement, his group's quantum cascade lasers have achieved lasing at milliwatt powers as low as $2.1 \mathrm{THz}(141 \mathrm{~mm})$ at a pulsed operating temperature of $137 \mathrm{~K}$. Reno's group is currently working to reduce the lasing threshold currents and increase the operating range toward room temperature.

VCSELs are promising sources for telecommunications wavelengths, but they generally display undesirable multiple transverse modes. K. Choquette's group (UIUC) demonstrated a single-transversemode quantum-well VCSEL with more than $3 \mathrm{~mW}$ of power whose transverse confinement is due to a periodic array of holes etched into the VCSEL surface. The holes form a 2D photonic crystal that supports only one transverse mode when a single hole position is left unetched (a "defect"). The group also demonstrated multilobed far-field laser patterns when several defects are present. The physics of the confinement is the same as that of photonic crystal fibers, although the scaled depth of the holes is much shorter than in the fibers and therefore does not provide as strong a transverse confinement.

Symposium Support: Hysitron, Inc.; JHU, Dept. of MSEE; and LLNL, CMLS Division.

\section{Crystal-Shape Control and Shape- Dependent Properties in Crystalline Materials Discussed}

\section{(See MRS Proceedings Volume 1087E)}

Symposium V on the shape control and shape-dependent properties of crystalline materials provided a comprehensive explanation of the shape-dependent evolution, stability, and properties of materials. The symposium offered an opportunity to see how similar synthetic stratagems applied to different materials can sometimes have strikingly different results, but in other cases can show an uncanny synergy that could not have been predicted based on simple scientific intuition.

Participants presented an array of crystal morphologies, ranging from simple platonic shapes to more complicated superstructures inspired by nature or industrious imaginations, all evidencing superior levels of synthetic control and advanced capabilities in characterization.
This was accompanied by theoretical studies offering both detailed explanations of shape-dependent phenomena and remarkable predictive capabilities. The symposium was not limited to any particular material and featured sessions on the shape of metallic, metal oxide, and semiconductor nanocrystals that covered much of the periodic table. The presentations covered studies on $0 \mathrm{D}, 1 \mathrm{D}$, and 2D structures and 3D networks and explanations of how the shape and dimensional anisotropy can be used to tailor highperformance applications in catalysis, sensing, optoelectronics, and biomedicine.

Symposium Support: Accelrys; American Scientific Publishers; FEI Co.; JEOL USA, Inc.; LLNL; NSF; Saint Gobain High Performance Materials; and Toyota Motor-Europe.

\section{Conjugated Organic Materials Highlighted in Field of Organic Semiconductors}

\section{(See MRS Proceedings Volume 1091E)}

The field of organic semiconductors is rapidly developing as advances and improvements in organic thin-film devices are driving the development of new technologies. Evidence for this was provided by Symposium AA on conjugated organic materials. The symposium was arranged into categories that covered many active areas of research, including nanoelectronic devices, organic PVs, organic FETs, and OLEDs.

G. Wallace (Univ. of Wollongong, Australia) spoke on the use of conjugated polymers for artificial muscles and nanobionics. His group is currently pioneering the use of conjugated polymers as electrical stimulants in nerve cell regeneration in bionic ears and damaged spinal cords.

Nobel laureate A. Heeger (UCSB) spoke about his recent work on high-performance thin-film solar cells. Using the low-bandgap polymer poly[2,6-(4,4-bis(2-ethylhexyl)-4H-cyclopenta[2,1-b;3,4-6']dithiophene)-alt-4,7-(2,1,3-benzothiadiazole)] (PCPDTBT) blended with C71PCBM in the presence of a small amount of alkanedithiol, power conversion efficiencies as high as 5.5\% were obtained.

Symposium Support: NSF, Sony Corp., and Universal Display Corp.

\section{Designer Biointerfaces Lead to Biomedical Applications} (See MRS Proceedings Volume 1093E)

Biologically relevant material surfaces impact a wide range of fields from drug delivery to implant technology. As such, the range of topics covered in Symposium $\mathrm{CC}$ on designer biointerfaces was diverse and multifaceted, illustrating the benefit of tailoring materials for specific applications. P.T. Hammond (MIT) discussed the use of electrostatic layer-by-layer assembly as a method for drug delivery from biomaterial coatings affording tunable simultaneous or sequential drug release. Work from J. Hubbell's group at Ecole Polytechnique Fédérale de Lausanne, Switzerland, showed that engineering the surface of nanoparticles with a collagen binding peptide identified by phage display imbued cartilage tissue targeting when injected intra-articularly in mice. S. Mitragotri (UCSB) provided evidence that the geometric shape of particles can influence drug release and that release is not mediated solely by surface area. Instead, phagocytosis of drug delivery particles can result in release, with the shape of a particle dictating the role of phagocytosis rather than the size of the particle.

Surface functionalization of supported bilayers (SBLs) was another focus. B. Ananthanarayanan (UCSB) described the use of lipid conjugated peptides in SBLs to create and screen multicomponent peptide surfaces. M. Mager (Stanford) introduced a method of lipid bilayer formation affording facile macroscopic patterning through lipid-coated air bubble collapse overcoming current limitations of the traditional methods of vesicle collapse and Langmuir-Blodgett deposition to create patterned lipid surfaces. K. Salaita (UC-Berkeley; LBNL) demonstrated the use of SBLs to determine how the biological activity of the ephrin-A1 ligand, which plays an important role in breast cancer biology, is dependent on its mobility. Immobility of this membrane-bound ligand limits membrane-protein clustering, which is necessary for downstream biological activity.

Symposium Support: Abbott Vascular and Medtronic CardioVascular, Inc.

\section{Progress Seen from Biological Materials to Biomimetic Materials Synthesis}

(See MRS Proceedings Volume 1094E)

Symposium DD provided an overview of new insights into the structure-function correlation in biological materials and the use of this information for the development of novel routes to synthesize materials with advanced properties. Investigations on the molecular mechanism of bacterial magnetite formation using targeted gene knockout (A. Komeili, UC-Berkeley) has already started to enable biotechnological approaches to using these biological structures for facile separations of macromolecules and cells (T. Yoshino and T. Matsunaga, Tokyo Univ. of Agriculture and Technology, Japan). Unique protein 
molecules from bacteria (S-layer proteins; D. Pum, Univ. of Natural Resources and Applied Life Sciences, Austria) and plants (SP-1; O. Shoseyov, Hebrew Univ. of Jerusalem, Israel) spontaneously self-assemble into highly regular $1 \mathrm{D}$ and 2D lattices. Introducing through genetic engineering binding sites for metal and semiconductor nanoparticles allows for the exploitation of these protein self-assembly processes for the formation of chains and 2D lattices of functional nanoparticles with controlled spacing. The group of $\mathrm{H}$. Waite (UCSB) investigated the cause for the exceptional hardness of certain biological materials (e.g., sandworm jaws and squid beaks) despite their low biomineral content. $\mathrm{Zn}^{2+}$ mediated cross-linking of histidine-rich proteins (e.g., sandworm jaws) and a novel biochemical cross-link, histidyl-dopa (e.g., squid beak), are the underlying principles for the mechanical stability of these minimally mineralized biomaterials. The findings open new design rules for connecting very soft and very hard materials in technological applications. The group of P. Messersmith (Northwestern) has developed a material that combines the structural features of gecko nanohair with the chemical adhesive principle of mussels, thus generating a seemingly universal glue, termed geckel, that sticks on all surfaces tested and shows only a small reduction of adhesiveness (i.e., $10-15 \%$ after more than 1000 adsorption-desorption cycles). Inspired by echinoderms, J. Aizenberg's group at Harvard University has combined "nanograss" made of high-aspect-ratio silicon pillars (or other materials) with hydrogels to generate novel rapid-response sensors for humidity, temperature, and other stimuli. The mechanism of dentin formation by amelogenin has been studied in vitro with the prospect of generating replacement materials with comparable mechanical performance (J. Moradian-Oldak, Univ. of Southern California). Emerging NMR data on the solution structure of amelogenin (K. Delak and J.S. Evans, New York Univ.) will greatly aid these studies. Constant-composition in vitro methods developed by G. Nancollas's group at the Univ. of Buffalo allow the various steps of dentin formation to be followed in vitro with unprecedented resolution.

Symposium Support: AFOSR, Air Force Research Lab., and LLNL.

\section{Frontiers of Research on Design and Synthesis of Responsive Biomaterials Reported}

(See MRS Proceedings Volume 1095E)

Symposium EE on responsive biomaterials for biomedical applications covered the frontiers of research on the design and synthesis of responsive biomaterials. Leading investigators reported recent progress on biomaterials that can respond to external, biologically compatible stimuli and their impact on various biomedical applications, especially drug delivery, tissue engineering, and sensing and diagnosis. K. Kataoka (Univ. of Tokyo, Japan), T.M. Reineke (Univ. of Cincinnati), T. Deming (UCLA), and T. Lodge (Univ. of Minnesota) reported the exciting progress in their laboratories on using responsive polymers and copolymers in drug delivery. J.A. Burdick (Univ. of Pennsylvania), T. Okano (Tokyo Women's Medical Univ., Japan), C.E. Schmidt (UT, Austin), and W.R. Wagner (Univ. of Pittsburgh) described recent progress in their research groups on utilizing responsive biomaterials in controlling cell behavior and in tissue engineering. S.I. Stupp (Northwestern) reported the discovery of dynamic selfassembly of bioactive structures. P. Messersmith (Northwestern) described the development of biomedical adhesives inspired by marine glues. D.N. Woolfson (Univ. of Bristol, UK) and R.V. Ulijn (Univ. of Manchester, UK) reported exciting progress on the development of peptidederived responsive nanomaterials. P. Stayton (Univ. of Washington) reported "smart" biohybrid materials for point-ofcare diagnostic devices. G. Wong (UIUC) presented recent developments in the understanding of the mechanisms of membrane-active antimicrobials and cellpenetrating peptides for drug delivery.

Symposium Support: AP Pharma; Boston Scientific Corp.; Cordis, a Johnson E Johnson Co. Medtronic Inc.; and Wyatt Technology Corp.

\section{Molecular Motors, Nanomachines, and Active Nanostructures Investigated (See MRS Proceedings Volume 1096E)}

One of the critical themes in the field of nanomachines is how to harness the motion in a useful device. Although this challenge is often described as the nanoto-macro divide, J.I. Zink (UCLA) gave a talk in Symposium FF on a device that acts at the nanoscale to control the delivery of chemicals from nanoporous silica. In the presentation, Zink outlined the approach of using the templated silica as a nanocontainer that employs molecular machines, in the form of rotaxanes, that can open and close the pore openings to release anything from dyes to drugs. New biocompatible rotaxane-based machines were attached around the rims of the pores to operate upon a change in $\mathrm{pH}$ encountered when they enter cells. Zink showed how the entry could be visualized by the release of dyes, suggest- ing the exciting opportunity to harness these devices for drug delivery. This potential and the related research conducted in collaborative teamwork have laid the foundation for the formation of a start-up company.

A vibrant session that was centered on active nanoscale systems built from DNA, proteins, or cells highlighted both historical roots and recent advances. Whereas N.C. Seeman (New York Univ.), J.N. Bath (Oxford, UK), and N. Pierce (Caltech) discussed DNA motors, a sequence of international speakers including D.V. Nicolau (Liverpool, UK), A. Mansson (Kalmar, Sweden), S. Diez (MPI-CBG Dresden), and T.Q.P. Uyeda (AIST, Japan) described the state of the art in engineering with motor proteins. G.D. Bachand (SNL) discussed the role of kinesin motor proteins in an active self-assembly process leading to the in vitro formation of microtubule spools. By combining transmission electron microscopy data with optical microscopy and a detailed statistical analysis, Bachand's team was able to shed light on the properties of these striking structures.

Symposium Support: AFOSR; Andor Technology; and Cytoskeleton, Inc.

\section{Progress Reported Toward Realization of Emerging Technologies of HTS} (See MRS Proceedings Volume 1099E)

Symposium II addressed progress toward the realization of the emerging technologies that incorporate hightemperature superconductivity through materials functionalization, nanoscale engineering, and creative processing. In the technology development front, $Y$. Chen presented the latest progress in the second-generation ( $2 \mathrm{G}$ ) wire program at SuperPower, Inc. He reported critical currents of 170 A per $\mathrm{cm}$ width over 935m-long MOCVD/MgO-IBAD 2G wire with high processing speeds. By improving multilayer processing and composition modification of the MOCVD thick film, critical currents exceeding $800 \mathrm{~A}$ per $\mathrm{cm}$ width at liquid nitrogen temperature of $77 \mathrm{~K}$ and self-field have been obtained on meter-length tapes. In addition, many intriguing studies toward realization of simplified, reduced-cost, high-performance coated conductor architectures have also been presented. In the flux pinning session, characterization and nanoscale engineering of coated conductors remain hot topics. K. Matsumoto (Kyushu Inst. of Technology, Japan) said that the in-field performance of coated conductors can be significantly enhanced by incorporating artificial pinning centers, such as $\mathrm{BaZrO}_{3}$ (BZO) and $\mathrm{Y}_{2} \mathrm{O}_{3}$ nanostructures, in the REBCO film. The irreversibility field, $B_{\text {irr }}$ 
and $J_{\mathrm{c}}(B)$ values at $77 \mathrm{~K}$ were found to be comparable to those of commercially available $\mathrm{NbTi}$ superconductors at $4.2 \mathrm{~K}$. S.-H. Wee (ORNL) presented the results of remarkably enhanced in-field orientation dependent properties of pulsed-laserdeposited (PLD) NbBCO films by introducing hybrid 1D-2D pinning structures, through combining columnar BZO nanorods and thin BZO interlayers. Wee said that it is possible to tune the in-field angular dependent anisotropy behavior of the PLD-REBCO films by controlling the structure of these artificial pinning centers. A comparative study has also been reported by P. Mele (Kyushu Inst. of Technology and CREST-JST, Japan) where both $\mathrm{BZO}$ and $\mathrm{BaSnO}_{3}$ (BSO) were incorporated into the REBCO films using the PLD method. TEM analysis reveals that the BSO nanoparticles are thinner and straighter than their BZO counterparts. Transport measurements on the BSO-doped sample showed a new record pinning force density of $28.3 \mathrm{GN} / \mathrm{m}^{3}$ $(77 \mathrm{~K}, \mathrm{~B} \| c)$, almost twice that of the BZOdoped sample. These and many other presentations highlighted advances in the synthesis and performance of both practical and scientifically important superconducting materials.

Symposium Support: American Superconductor; ANL; Kyushu/West-Japan Branch of Cryogenic Association of Japan; LANL; ORNL; SuperPower, Inc.; and SWCC Showa Cable Systems Co.

\section{Materials Challenges Explored for Electrical Storage \\ (See MRS Electronic Proceedings Volume 1100E)}

Efficient utilization of electricity generated from intermittent renewable energy sources, such as solar or wind, requires electrical energy storage (EES). Furthermore, vast improvements in EES are needed to enable the reliable and safe storage of electrical energy for transportation, commercial, and residential applications. The performance of current EES systems is limited by the performance of materials. The initial presentations in Symposium JJ focused on the critical issues and approaches needed to improve materials in batteries and electrochemical capacitors (ECs). The symposium was inspired by a recent workshop sponsored by the DOE Office of Basic Energy Sciences entitled "Basic Research Needs for Electrical Energy Storage" (www.sc.doe.gov/bes/ reports/list.html) and included overviews provided by key workshop participants. S. Whittingham (Binghamton Univ.) discussed the critical need for new materials to enable the development of future EES systems. S.-J. Visco (LBNL) discussed fun-

\section{A. Paul Alivisatos Delivers Kavl Lecture on Nanoscience}

In his Fred Kavli Distinguished Lecture in Nanoscience, A. Paul Alivisatos of the University of California-Berkeley and Lawrence Berkeley National Laboratory described work on nanocrystals accomplished by his group over the past several years, including some very recent results. His talk was divided into three parts. In the first part, he described the use of nanocrystals and DNA to build artificial nanocrystals, describing the example of a plasmon ruler using these new structures. DNA was used to couple two or more inorganic nanoparticles, which occurs

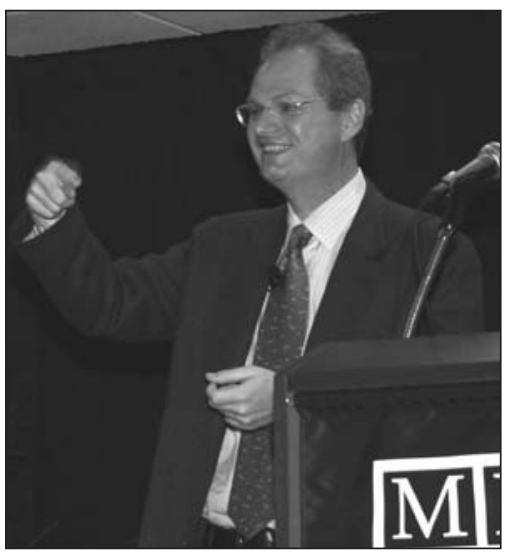
through plasmons. Quantum dot-gold nanoparticle aggregates can thus be formed with one or more $\mathrm{Au}$ nanoparticles attached through DNA linkers. It should be noted that the DNA is not rigid, but rather is flexible, and hence the distance cannot be specified, Alivisatos said. This leads to a very interesting plasmon coupling phenomenon, he said. By pairing Au or Ag particles, for instance, light scattering changes drastically, with larger scattering from two particles that is easily visible. Alivisatos provided an example of using this phenomenon to detect cleavage of DNA attached to two or more particles by an enzyme.

In the second part of his talk, Alivisatos described how chemical transformations can be used to obtain complex nanocrystal molecules. His group, as well as others, has demonstrated how very complex shapes of colloidal inorganic nanocrystals are possible through sequential chemical operations. He described the examples of cation exchange to change the physical and chemical properties of the nanocrystals, as well as forming a nanoparticle within another and forming branched nanoparticles. These processes can be performed sequentially in any order. In an example, CdSe underwent rapid cation exchange upon addition of $\mathrm{Ag}^{+}$to form $\mathrm{Ag}_{2} \mathrm{Se}$, which, through addition of $\mathrm{Cd}^{2+}$, was completely converted back to CdSe. Such a reversible transition is not possible in the bulk material. Moreover, the volume of the nanoparticle was completely preserved. Alivisatos also described cation exchange in semiconductor nanorods with shape preservation, as well as in complex nanostructures such as hollow spheres and tetrapods. He said that, so far, it has been very difficult to predict the structures obtained in these nanoparticle transformations. He also described the evolution of spaced dots on a rod into segments by ripening and intercalation.

In the third and final part of his Kavli Lecture, Alivisatos described new tools for probing nanocrystals. One is a method for in situ observations of nanocrystal motion in water in a transmission electron microscope using a liquid cell. Trajectories of $\mathrm{Au}$ nanorods showed two distinct length scales of motion, outwardly similar to Brownian motion, with mostly localized movement followed by a large jump. The surprising aspect of this process is that the particles appear to roll during the long jumps, as suggested by TEM observations.

The Kavli Lectureship is awarded by the Kavli Foundation, which supports scientific research, honors scientific achievement, and promotes public understanding of scientists and their work. Its particular focuses are astrophysics, nanoscience, and neuroscience.

damental challenges in the development of electrolytes and anodes in advanced battery systems. J.R. Miller (JME, Inc.) discussed electrochemical capacitors, including the significance of electric double-layer charge storage and critical technology needs. M.M. Thackeray (ANL) focused on battery anodes and cathodes and the need to understand, characterize, and develop new energy storage chemistries. B. Dunn (UCLA) noted that capacitive storage of electrical energy is receiving increasing interest because of potential opportunities in large-scale energy regeneration and energy storage technologies and discussed the scientific bottlenecks facing this technology. A.A. Gewirth (UIUC) discussed cross-cutting issues relevant to research in 
EES, including charge transfer at interfaces, new measurement tools and techniques, spectroscopy and structure, electrolytes, and large-scale computation. Following these presentations were talks and posters from a large number of research laboratories from around the world. These reports covered recent advances in the development of new materials for both batteries and ECs, including characterizing and modeling materials behavior in the operation of EES.

\section{Strategies Investigated for Improvement of Light Coupling in PVs (See MRS Electronic Proceedings Volume 1101E)}

The strongly growing PV market (over $20 \mathrm{GW}$ annual module production capacities foreseen for 2011) will need continuous scientific and technological input to become a significant part of the 21st century electricity supply. Symposium KK reviewed state-of-the-art approaches for improving the efficiencies of PV devices, with a focus on strategies to improve the light coupling in the solar cells.

Among the highlights reported were light-trapping strategies for thin-film siliconbased solar cells. The production solutions for such devices are now available, and the installed annual production capacity is expected to reach $4 \mathrm{GW}$ by the end of 2010. T. Oyama (Asahi Glass Co., Ltd., Japan), whose company is a leader in the production of large plates of high-quality nanostructured oxides on which such solar cells are fabricated, presented recent results for TCO with double structures at both micrometer and nanometer size that could enable significant increases in the efficiency of the solar modules. The use of various grating structures to couple the light more effectively were presented by several groups, with convincing results illustrating the effectiveness of the approach both experimentally and theoretically (F.-J. Haug, Univ. Neuchâtel, Switzerland; H. Stiebig, FZ Jülich, Germany; R.H. Morf, PSI, Switzerland; J. Krc, Univ. Ljubljana, Slovenia; and H. Sai, AIST, Japan). Surfaceplasmon-induced losses in textured backreflectors were presented and analyzed by different groups, and strategies for avoiding or minimizing their absorption losses were discussed.

Conversely, other scientists reported on enhanced light scattering by localized plasmonic effects in metallic nanoparticles that yielded increased light absorption in various devices (K.R. Catchpole and F.-J. Beck, ANU, Australia) and possibili-

ties of current increase by surfaceplasmon-active silver nanohole films were described (T.H. Reilly, NREL).

Original detailed calculations of the electronic properties of coupled quantumwell solar cells were presented by U. Aeberhard (PSI, Switzerland), whereas review talks by A. Marti (Univ. Politécnica de Madrid, Spain) and A.G. Norman (NREL) demonstrated the potential of intermediate-bandgap solar cells but also the difficult challenge of demonstrating device improvement, compared to conventional devices. The latest results on the effects of multiple exciton generation were presented by M.C. Beard (NREL), whereas some controversial results were reported by J.P. Nair (MIT).

A number of presentations reviewed the potential, but also the challenges, of alternative approaches such as the use of up-conversion effects (G. Conibeer, UNSW, Australia) using rare-earth elements, whereas F.N. Castellano (Bowling Green State Univ.) presented results for low-power noncoherent upconversion in solid polymer films.

Symposium Support: Kaneka Corp., Nuon Heliantos, and OC Oerlikon Balzers.

\section{Materials and Devices Explored for Direct Energy Conversion (See MRS Proceedings Volume 1102E)}

Symposium LL on energy harvesting combined solar, thermoelectric, magnetic, and vibrational approaches, often with several techniques being used in the same device. The symposium was kicked off by Nobel laureate A. Heeger (UCSB) who discussed electrochemical doping in an electrolyte-gated polymer transistor. Heeger showed how high current densities and efficient light emission can result from these devices. In particular, the possibility of powering these devices using energy-harvesting technologies on the same platform was discussed in the question session. Next, L. Hammarstrom (Uppsala Univ., Sweden) presented a talk entitled "Hydrogen from Sun and Water-From Natural to Artificial Photosynthesis." The idea of increasing the efficiency of devices by using active biological pathways without needing the biological framework is not new, but Hammarstrom focused on the substantial progress that has been made. In the area of thermoelectric materials, C. Dames (UC-Riverside) discussed the broad range of phonon mean free paths (MFPs) important for heat conduction. Whereas most discussions have focused on a single, average value of the bulk MFP, Dames showed that phonons with long MFPs are more important for thermal conductivity than is 
commonly appreciated. For example, in common thermoelectric alloys such as $\mathrm{PbTe}$ and $\mathrm{Sb}_{2} \mathrm{Te}_{3}$ at room temperature, conventional estimates for the bulk MFP are $10 \mathrm{~nm}$ or less. Analysis of these materials has shown that approximately $25 \%$ of the heat is carried by MFPs longer than $100 \mathrm{~nm}$, with over $10 \%$ of the heat carried by MFPs longer than $500 \mathrm{~nm}$. This "long tail" of phonon MFPs has favorable implications for the design of materials with low thermal conductivity. In the area of mechanical energy harvesting, G. Carman (UCLA) discussed a broadband kineticenergy-harvesting device based on frequency rectification. Carman used a piezoelectric material to convert ambient mechanical vibration into electrical energy effectively while off-resonance, relying on frequency rectification. Frequency rectification is the process of taking largeamplitude displacements at relatively low frequencies and upconverting the signal to low-amplitude displacements at high frequency. This dramatically reduces the size of the kinetic-energy-harvesting device required in energy-rich environments and eliminates the resonant tuning required in current devices.

Symposium Support: LLNL.

\section{Relationship between \\ Physicochemical Properties of Nanomaterials and Their Impact on Health and Environment Explored (See MRS Proceedings Volume 1103E)}

Nanoscale materials show a high degree of tunability with respect to their physical and chemical properties. As materials researchers exploit this tunability to create flexible and adaptive materials for commercial applications, it becomes critical to assess the potential impact that these novel materials might have on organisms and the environment.

To address these health and safety questions, several researchers presented technologies in Symposium MM that characterize the biological response to nanomaterials in vitro, both quantitatively and in real time. Examples of such technologies include single-cell analysis, an approach that employs a nondestructive nanoprobe to assess the intracellular response of a single cell (T. Vo-Dinh, Duke Univ.), and bioRaman, an in situ method to investigate intracellular biomolecule-nanoparticle interactions (G. Pyrgiotakis, Univ. of Florida). Technologies capable of assessing environmental fate and transport include a biomimetic assay that simulates an oxidative lingolytic fungal soil environment (K.M. Metz, Univ. of Wisconsin-Madison) and genomic analysis of daphnia following aquatic exposure to nanomaterials

\section{Michael S. Strano Gives Award Presentation on Chemistry of SWNTs}

Michael S. Strano of the Massachusetts Institute of Technology, recipient of the 2008 MRS Outstanding Young Investigator award, presented an award talk on the chemistry of single-walled carbon nanotubes. He described the use of SWNTs as tunable near-infrared fluorescent biological sensors. One of the major advantages is the non-photobleaching characteristics of SWNTs with stable photoemission in the near-IR region of the spectrum. Interestingly, the near-IR region is situated in the transparency window for biological tissue. When a specific DNA molecule was wrapped around the SWNTs, the transition of the DNA secondary structure from one conformation to another was found to modulate the dielectric environment of the SWNTs

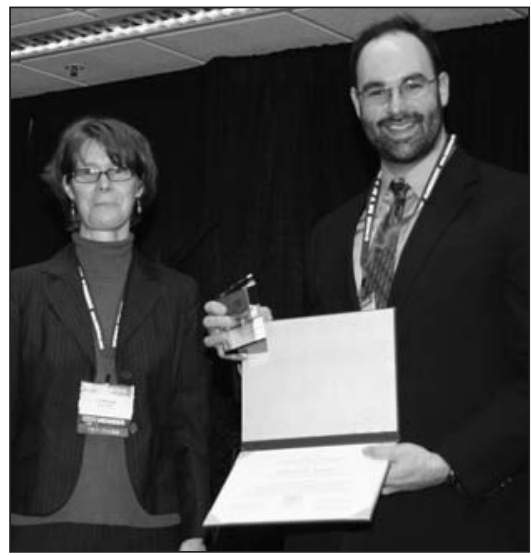

Michael S. Strano of MIT (right) receives the 2008 Outstanding Young Investigator Award from MRS President Cynthia A. Volkert (Univ. of Göttingen).

around which it was adsorbed. The SWNT bandgap fluorescence underwent a red shift when the nucleotide oligomer was exposed to counterions that screened the charged backbone. This was then used as a biosensing technique to detect mercuric ions in living mammalian cells. Strano also described the use of SWNTs with glucose oxidase immobilized on them as $\beta$-D-glucose sensors that can be implanted under the skin to monitor glucose levels. Another area being explored for these materials is as a hydrogen peroxide sensor using functionalization with ferricyanide.

Strano also discussed the selective covalent chemistry of SWNTs. The goal of this work is to be able to predict the reactivity for a given $(n, m)$ nanotube and to be able to separate and sort SWNTs by electronic structure: metallic and semiconducting. Previously, selectivity was limited to geometric structure. Strano's group used Raman and photoluminescence spectra collected in situ, revealing a series of two first-order reactions involving an adsorbed intermediate and covalently bound product. A 4-hydroxybenzene diazonium reagent was used to selectively separate metallic and semiconducting SWNTs. The range of Strano's work with SWNTs provides a glimpse of the versatility of the material and a bright outlook for more surprises to come in the future.

The Outstanding Young Investigator Award was established to recognize outstanding interdisciplinary materials research by a young scientist or engineer.

(R. Klaper, Great Lakes WATER Inst.). The research capabilities of the Molecular Foundry (M. Alper, LBNL) were discussed as an example of the DOE nanomaterials synthesis and characterization facilities. These laboratories are open to collaboration with investigators from industry and universities.

Tuning the biological response to nanomaterials is a complex and multifactorial challenge. Material components that affect biology include synthesis byproducts, surface chemistry, and aggregation state (M. Wiesner, Duke), as well as size, shape, and crystal phase. The extent and type of surface derivitization and solubility also contribute to the cellular and molecular response (V. Colvin, Rice). The safety of pegylated coatings was challenged (N. Lewinski, Rice), and genotoxicity of metal oxides in vitro were reported to be weak (M. Carriere, CEA Saclay, France). Proteomics analysis was used to identify size-dependent differences in cellular pathway activation (F. Chen, LBNL), and a comparison of carbon nanotube biology to asbestos biology demonstrated similarities in cellular toxicity (R. Duffin, Queen's Medical Research Inst., UK).

\section{Electronic Structure Calculations in Actinides IV Remain a Challenge} (See MRS Proceedings Volume 1104)

A central theme of Symposium NN was the need to understand and control the response of $5 f$ electrons to their environment, a theme that cut across disciplines ranging from nuclear fuels and actinide waste disposal and storage to molecular chemistry and from plutonium's equation 
of state and metallurgy to complex states that arise from strong electronic correlations. Progress is being made in each of these areas, but electronic structure calculations, which underlie several of these disciplines, remain a particular challenge. This fact stood out most obviously in band calculations for elemental $\mathrm{Pu}$ and many of its compounds. As discussed in several presentations, conventional approaches to band calculations based on the local spin density approximation, including Coulomb correlations, often lead to inconsistencies with measured properties, such as significant differences between calculated and measured paramagnetic moments in a variety of actinide materials including elemental Pu. The problem lies in developing realistic models of strong correlations. Recent advances in a new approach, called dynamical mean field theory, hold substantial promise in this regard and, as discussed by G. Kotliar and collaborators at Rutgers University, have been used to account for prominent features in angleintegrated photoemission spectra of $\mathrm{Pu}$ and of other strongly correlated actinides. These calculations often predict a timeaveraged nonintegral $5 f$ configuration, with an $f$ occupancy in Pu-based systems ranging from somewhat over 5 to nearly 6 , depending on the particular material, implying that mixed-valence systems are more common than previously thought. Because the valence configuration has implications for understanding chargetransfer processes in molecules, the reactivity of actinides with their surrounding atmospheres, and both equations of states and unconventional superconductivity found in some $\mathrm{Np}$ - and Pu-based intermetallics, it is especially important that electronic structure calculations be pushed to new limits of predictability and that they be benchmarked against new experiments, such as angle-resolved photoemission, de Haas-van Alphen measurements of Fermi surface topology, and inelastic neutron scattering, that are sensitive to spin fluctuations, which are carried by fluctuations of the valence.

Symposium support: LANL, LBNL, LLNL, and the Actinide Steering Committee (LLNL).

\section{Business Aspects of Nanotechnology Revealed}

(See MRS Proceedings Volume 1106E)

Symposium PP on "The Business of
Nanotechnology" offered presentations in areas ranging from energy, electronics, and biotechnology to environment. A.C. Smith (Lehmann Brothers) and C. Gay (Applied Materials) discussed the business of solar energy and electricity for the 21st century. M. Blohm (General Electric) and C. Liu (Cooley Godward Kronish LLP) treated the issues of nanotechnology commercialization and patent strategy, respectively. V. Colvin (Rice Univ.) and A. Vaseashta (Marshall Univ.) addressed the issue of environment while K. Gonsalves (Univ. of Charlotte, North Carolina) focused on nanobiotechnology. H. Hahn (Institute for Nanotechnology, Germany) gave an interactive presentation on the mechanics of creating a start-up company. A. Gandhi (Applied Materials) and S. Giordani (TTP Lab.) moderated a forum entitled, "How to Start and Grow a Successful Start-Up Company: A Survival Kit for Entrepreneurs." Panelists included venture capitalist M. Beckmann (Nanostart, Germany), lawyer L. King (Skadden LLP), and investment manager Smith of Lehmann Brothers.

Symposium support: Applied Materials, Inc.; General Electric Company; Sage Publishing Co.; and The Nanotechnology Institute.

\section{MRS Elects Officer, Board of Directors for 2009}

Members of the Materials Research Society have elected one officer and five directors to join the 2009 MRS Board of Directors. The board is composed of the officers and up to 18 directors. The officers of the Society are the president, the vice president (who is also the president-elect), the secretary, the treasurer (a position appointed by the Board of Directors), and the immediate past president. The annual election ended September 26, 2008.

The Board of Directors is organized into the following governing committees: Planning, Operational Oversight, External Relations, and Governance. The president, who serves as chair of the board, appoints each of the directors and officers to one of the first three governing committees, and designates the chairs of these committees.

Terms of office expire at the end of the year indicated in parentheses. The asterisk $\left(^{*}\right)$ designates those who are newly elected or appointed.

\section{MRS Officers}

President

Shefford P. Baker

Cornell University
Immediate Past President

Cynthia A. Volkert

University of Göttingen

Vice President (President-Elect)

* David S. Ginley

National Renewable Energy Laboratory

Secretary

Bethanie J.H. Stadler

University of Minnesota

Treasurer

* Linda L. Horton

Oak Ridge National Laboratory

\section{Board of Directors}

J. Charles Barbour (2009)

Sandia National Laboratories

* Eberhard Bodenschatz (2011)

Max Planck Institute for Dynamics and Self Organization

Ian W. Boyd (2010)

University College London

Yves J. Chabal (2010)

University of Texas at Dallas

*Jim De Yoreo (2011)

Lawrence Berkeley National Laboratory
Susan P. Ermer (2009)

Lockheed Martin Advanced

Technology Center

Mary E. Galvin (2010)

Air Products and Chemicals, Inc.

Charles C. Han (2010)

Chinese Academy of Sciences

Gregg S. Higashi (2010)

Applied Materials, Inc.

Cherie Kagan (2009)

University of Pennsylvania

* Christine A. Orme (2011)

Lawrence Livermore National

Laboratory

* Michael F. Rubner (2011)

Massachusetts Institute of Technology

*Takao Someya (2011)

The University of Tokyo

James C. Sturm (2009)

Princeton University

Richard A. Vaia (2010)

U.S. Air Force Research Laboratory

Timothy P. Weihs (2009)

Johns Hopkins University 